\title{
UNA ESTIMACIÓN DEL COSTE DE LA VIDA EN ESPAÑA, 1861-1936*
}

\author{
ESMERALDA BALLESTEROS DONCEL \\ Universidad Carlos III de Madrid
}

\section{RESUMEN}

En el siguiente artículo se presenta la estimación de un índice del coste de la vida en España entre 1861 y 1936 basado en la reconstrucción de series de precios relativas a doce provincias de la geografía peninsular. La autora describe el proceso de cuantificación detallando la naturaleza de las fuentes, los bienes seleccionados y la ponderación aplicada a los mismos. Aunque los resultados no modifican en el medio y largo plazo nuestro conocimiento sobre la dinámica de la economía española, sí sugieren una lectura renovada sobre la intensidad de las crisis de subsistencias en la segunda mitad del novecientos.

In this essay is presented an estimation of a cost of living index in Spain for the period 1861-1936, based on the reconstruction of series of prices for twelve Spanish provinces. The author describes the quantitative process, detailing the nature and

* Este artículo es parte de mi tesis doctoral en la que abordo el estudio de los niveles de vida en España en los siglos XIX y XX. La investigación se ha beneficiado de una Beca Predoctoral del Ministerio de Educación y Ciencia, adscrita al Departamento de Economía de la Universidad Carlos III. Quisiera expresar mi agradecimiento por el aliento recibido de mis compañeros en Getafe y, de forma particular, a los profesores Leandro Prados de la Escosura y James Simpson. La idea de escribir este artículo y el interés por aprender el oficio se deben al permanente estímulo de D. S. Reher; su orientación y su paciencia han hecho posible esta contribución. Deseo agradecer también a Antonio Santamaría García, Tomás Martínez Vara, un evaluador anónimo y algunos miembros de la Secretaría de Redacción de la $R H E$, las observaciones que hicieron sobre el primer manuscrito. Naturalmente, los posibles errores son de mi exclusiva responsabilidad. 
origin of the sources, the goods selected and the budget criteria. Although the results do not modify our knowledge about the dynamics of the Spanish economy, they suggest a new reading intensity of subsistence crisis in the second half of ninenteenth century.

\section{INTRODUCCIÓN}

El conocimiento de los precios ha constituido y constituye un objetivo central de análisis para la comunidad científica internacional de historiadores económicos, dado que en ellos se sintetizan numéricamente aspectos generales de la economía de un país. Su investigación permite plantear hipótesis en relación a distintos problemas (secuencia de los ciclos económicos, integración de mercados, intensidad y alcance de las crisis, etc.) y mediante su observación y examen estadístico se pueden obtener importantes respuestas.

En el marco general en el que se inserta nuestra investigación, esto es, el estudio de los niveles de vida en España en los siglos XIX y XX, el interés por los precios resulta básico y prioritario. Uno de los enfoques posibles para evaluar el bienestar material de los individuos consiste en averiguar a qué bienes y servicios se puede acceder con un determinado nivel de renta. Lamentablemente no se ha publicado en España, por el momento, ninguna serie económica que responda a esta cuestión, por lo que hemos procedido a construir una estimación del coste de la vida a partir de la recreación de doce índices provinciales de precios para el período que transcurre entre 1861 y 1936.

En las siguientes páginas se detallan los criterios y materiales empleados en la estimación así como las limitaciones en que incurre el cálculo. A continuación se examina el comportamiento de la serie analizando la incidencia de las crisis de subsistencia. Y finalmente se establecen comparaciones entre nuestra estimación del coste de la vida e índices de similares características disponibles para dos ciudades españolas (Barcelona y Madrid) y algunos países europeos de nuestro entorno (Portugal, Italia, Francia e Inglaterra).

\section{CONSIDERACIONES SOBRE LA CONSTRUCCIÓN DE INDICES GENERALES DE PRECIOS}

El interés y conocimiento sobre la Historia de los Precios en Europa es hoy bastante completo en virtud del gran número de publicaciones que se 
centran en su estudio. En España, por el contrario, existe un claro desfase entre la riqueza documental proporcionada por Hamilton para la Edad Moderna y el relativo vacío estadístico de la Edad Contemporánea. Con las series estadísticas que se aportan en este artículo se reduce en parte esta laguna pero no se resuelven todas las carencias, particularmente llamativas para la primera mitad del novecientos. Antes de acometer el objetivo del trabajo se propone un breve recordatorio sobre los problemas que envuelven a la construcción de índices generales de precios.

Es posible diseñar una caracterización ideal previa a la localización y explotación del material. Resulta además eficaz y recomendable hacerlo, dado que con el cotejo entre lo exigido a la información y lo realmente hallado se puede determinar el alcance de la estimación. Los requerimientos básicos para confeccionar un índice del coste de la vida giran en torno a tres ejes: naturaleza de los precios, elección de los bienes de consumo y ponderación de los mismos.

Con respecto a la naturaleza de los precios, para construir un índice del coste de la vida se aconseja el empleo de los precios de mercado al por menor. Estas cotizaciones expresan las cantidades efectivamente pagadas por los consumidores finales al incluir los costes derivados tanto de la producción y el transporte como de la aplicación de impuestos indirectos. Esta valiosa información se custodia generalmente en archivos locales y en ocasiones también se publica periódicamente en prensa, pero su alcance queda restringido a un área local por lo que su utilización en un análisis de ámbito nacional es arriesgado.

Las alternativas documentales a los precios al por menor son dos. Por un lado, los precios al por mayor expresan el valor de producción de los bienes, por lo que podemos definirlos como cotizaciones brutas. En muchas ocasiones se han empleado como base para la confección de índices del coste de la vida y sus resultados se han aceptado asumiendo la hipótesis de que los costes derivados de la comercialización de los bienes se mueven en la misma dirección ${ }^{1}$. Por otro ládo, contamos con los precios institucionales, que emanan de los establecimientos que requieren abastos para el cuidado de grupos de población a su cargo, tales como hospitales, internados, cuarteles, prisiones o centros eclesiásticos. El problema de este material es que las cantidades reseñadas puedan ser objeto de contrato anticipado y no reflejen el movimiento natural de los precios ${ }^{2}$.

M. W. Flinn (1974), p. 402.

2 P. Deane y W. A. Cole (1962), p. 13. 
La historiografía británica cuenta con estimaciones realizadas con precios de los tres tipos descritos. Los índices de Jevons (1895), Silberling (1923), Kondratieff (1928), Rousseaux (1938) y Gayer, Rostow y Schwartz (1953) se sustentan en precios al por mayor (wholesale prices). El trabajo de Tucker (1936) está basado en precios institucionales (contract prices) procedentes de la contabilidad de tres hospitales londinenses. Mientras que las series de Phelps Brown y Hopkins (1956) y Lindert y Williamson (1980, 1983 ) poseen un caracter mixto: se nutren de precios al por mayor para algunos productos y períodos y de precios institucionales para otros. Tan sólo Wood creó en 1899 un índice del coste de la vida con precios al por menor (retail prices) para 1790-1860, y recientemente Feinstein ha publicando un nuevo índice de ámbito nacional tomando como base principal precios al por menor ${ }^{3}$.

La historiografía española también cuenta con series de precios elaboradas a partir de cotizaciones de distinta naturaleza. Con respecto a los precios institucionales, el trabajo más representativo es el de Hamilton (1934). Las series de precios al por mayor más utilizadas para el siglo XIX son las publicadas por Sardá (1948), y en cambio se valen de precios al por menor las estimaciones de Reher y Ballesteros (1993a), Pérez Castroviejo (1992) y Pérez Sánchez (1996).

En un plano secundario hay que valorar las características de periodicidad y localización de los registros. Atendiendo a la frecuencia con que se suministran los precios se pueden encontrar cotizaciones diarias, semanales, mensuales y anuales. La mejor elección es reflejar la información más detallada y proceder posteriormente a la agregación de datos en función de las hipótesis de trabajo. En cuanto a la localización de las series, la clasificación de precios locales, regionales o nacionales está vinculada a la naturaleza de la estadística y la calidad de las fuentes debe ser examinada por el investigador que las considere.

Un aspecto de primer orden, y escasamente planteado en el diseño previo a la estimación de un índice general de precios, es el relativo a la homogeneidad de los productos que lo integran. La calidad, composición y posible adulteración de los artículos de consumo son cuestiones que se deben tener en cuenta, porque el asunto puede llegar a ser dramático. Hay que recordar que Sánchez-Albornoz y Carnero Arbat no publicaron las series provinciales del vino en España entre 1860 y 1890 por esta razón:

${ }^{3}$ Ver Ch. Feinstein (1995), pp. 9-32. 
[... la heterogeneidad del producto no sólo prohíbe promediar o buscar cualquier expresión común al conjunto de las series; también desaconseja comparar trayectorias. Decididamente, las series oficiales del vino no son homologables] ${ }^{4}$.

Las controversias suscitadas en torno a la naturaleza de los precios guardan una íntima relación con la disponibilidad y el estado de las fuentes. De hecho, si existieran estadísticas completas de precios al por menor este aspecto no tendría apenas relevancia. La cuestión de peso es cómo se resuelve en la investigación una laguna en los datos. La alternativa de tener que recurrir a precios, sean de la producción, institucionales, incluso de las importaciones, puede suponer una difícil decisión.

La elección de los bienes que habrán de formar parte del índice del coste de la vida y su ponderación representan una cuestión central. Determinar la composición de la cesta de la compra implica conocer, a grandes rasgos, las preferencias y los hábitos de consumo de la población. Tradicionalmente, este aspecto se ha deducido a partir de la recopilación y análisis comparado de los presupuestos familiares. En nuestro caso, hemos localizado 59 testimonios que describen la distribución media del gasto en España entre 1850 y $1920^{5}$. A partir de este material podemos afirmar que el desembolso principal declarado por las familias se destinaba a la alimentación, en torno a un 70 por ciento. Su importancia contrasta con el reducido número de artículos en la ingesta: pan, patatas, legumbres, tocino, aceite; siendo el vino y el aguardiente los principales complementos calóricos. El restante 30 por ciento se reparte de manera homogénea entre la vivienda, el vestuario y el apartado de otros gastos.

Existe una cierta similitud entre la estructura de gastos descrita para España y los presupuestos británicos disponibles para el novecientos, tanto las partidas como su magnitud coinciden. Por poner un ejemplo, los coeficientes de ponderación aplicados por Williamson en su estimación clásica del coste de la vida entre 1781 y 1850 son: alimentos, 63,8 por ciento; alquileres, 16,6 por ciento; combustible y alumbrado, 6,6 por ciento y ropa, 13,0 por ciento ${ }^{6}$. Las

${ }^{4}$ N. Sánchez-Albornoz y T. Carnero Arbat (1981), p. 29; también el Grupo de Estudios de Historia Rural adoptó esa misma decisión GEHR (1981b).

Este material de investigación se describe con profusión en un capítulo de mi tesis doctoral ( Niveles de vida en España, siglos XIX y XX»). Las conclusiones y resultados de estos 59 presupuestos pueden consultarse en la comunicación enviada al III Seminario sobre Economias y Estrategias Familiares con el título «El presupuesto familiar como indicador de los niveles de vida (1850-1991)», celebrado en la Universitat Pompeu Fabra del 3 al 5 de marzo de 1997.

6 J. G. Williamson (1987), pp. 277-298. 
divergencias se producen en nuestro siglo, ya que si se examinan los resultados de las Encuestas de Presupuestos Familiares disponibles desde 1958, se aprecia una gran estabilidad en la distribución de gastos de los hogares españoles, que comienza a modificarse de forma significativa a finales de los años setenta.

\section{CRITERIOS ADOPTADOS EN LA ESTIMACION DEL ÍNDICE DEL COSTE DE LA VIDA EN ESPAÑA, 1861-1936}

\subsection{Selección de una muestra}

Nuestro interés por la recopilación y análisis de los precios está vinculada a su aplicación al estudio de los niveles de vida. En este sentido, no era necesario reconstruir una exhaustiva estadística que abarcara la totalidad de la geografía española, por lo que decidimos seleccionar un estratégico grupo de provincias a partir del cual cupiera promediar un valor de conjunto. Decidimos utilizar el salario real como variable discriminante, por lo que calculamos una razón entre precios y salarios que nos permitiera, grosso modo, explorar la geografía del bienestar. Ese cociente se calculó para la totalidad de las provincias peninsulares en los años 1860 , 1896,1915 y $1930^{7}$.

$\mathrm{La}$ información relativa a los precios contiene las cotizaciones provinciales de trigo en los dos cortes de la segunda mitad del XIX y el precio del pan en los años 1915 y 1930. Para los salarios se localizaron en los cuatro años señalados los jornales de peones ordinarios, tanto agrícolas como no agrícolas, de cuya media se obtiene el valor salarial. Para poder establecer la comparabilidad entre los datos se calcularon índices estableciendo como base el promedio de las 47 provincias ${ }^{8}$.

Los resultados de los cocientes permiten distinguir tres grupos de provincias según posean índices superiores, inferiores o próximos al valor promedio. Cataluña, la costa del Cantábrico y Madrid tienen razones superiores a 100; es decir, la relación entre niveles salariales y precios resulta favorable. Galicia, Extremadura, Andalucía oriental y el sur levantino presentan co-

7 La eliminación de Baleares y Canarias responde a las lagunas sistemáticas que presentan los datos de ambas provincias para el conjunto de las 12 variables que han servido para la selección de la muestra.

${ }^{8}$ Los datos e índices pueden consultarse en el apéndice estadístico, tablas 1-4. 
cientes inferiores al nivel medio, en torno al cual se encuentran Aragón y las dos Castillas. Finalmente, se eligieron de cada grupo cuatro provincias, resultando seleccionadas Barcelona, Madrid, Sevilla y Vizcaya como unidades con alto nivel de vida. En un estadio intermedio se optó por La Coruña, Navarra, Valladolid y Zaragoza, y como exponentes de las provincias con bajo nivel de vida se seleccionaron Alicante, Cáceres, Granada y Toledo.

Aunque los criterios de selección pueden ser discutidos, la muestra obtenida representa una cuarta parte de la geografía española y en ella creemos que queda reflejada la heterogeneidad social y económica del país.

\subsection{Fuentes}

Es espectacular el retraso con el que la Administración española acometió la formación de una estadística moderna de precios de consumo, que no alcanzó una adecuada organización hasta los años cuarenta de nuestro siglo.

No existe ninguna fuente que centralice de forma seriada una estadística de precios en el período que transcurre entre la segunda mitad del siglo XIX y el primer tercio del siglo XX. Ello obliga a encadenar los datos combinando distintos materiales, con lo que nuestro índice se descompone en tres subperíodos: 1861-1890, 1891-1906 y 1907-1936.

En el primer subperíodo 1861-1890 nos hemos servido de la estadística de precios elaborada por el Ministerio de Fomento y que fue publicada simultáneamente en los Boletines Provinciales y en La Gaceta de Madrid'. Dicha estadística recoge las cotizaciones mensuales por provincias de catorce artículos de consumo primario: trigo, cebada, centeno, maíz, garbanzos, arroz, aceite, vino, aguardiente, carnero, vaca, tocino y paja de trigo y de cebada.

Para nuestro análisis se han considerado sólo ocho artículos. La renuncia a incluir cebada, centeno y maíz responde a que aunque se tiene la certeza de que la población, de forma particular los sectores más desfavorecidos, adquirían y elaboraban pan de distinta composición, no disponemos de

${ }^{9}$ N. Sánchez-Albornoz ha sido, hasta ahora, el único investigador que ha construido sobre una parte de estos datos una estadística de precios en España en la segunda mitad del siglo XIX, detallando el proceso legal y las inquietudes políticas a las que responde esta valiosa información (1975), pp. 3-11. 
parámetros fiables sobre su importancia en el consumo total. Además, las diferencias geográficas en la producción de cereales panificables también influyen sobre los patrones de consumo, pero, hoy por hoy, sólo tenemos algunas medidas indirectas que impiden, a nuestro juicio, establecer con certeza tales diferencias ${ }^{10}$.

Prescindimos del carnero por ser una variedad de carne no siempre consignada en las estadísticas, ya que hemos pretendido mantener una selección de productos lo más homogénea posible. La eliminación de la paja obedece a que se trata de un producto destinado al ganado. Los cambios en las medidas, monetarias y metrológicas, nos ha obligado a homogeneizar las series en pesetas y en unidades del sistema métrico decimal.

Para el segundo subperíodo 1891-1906 no hemos localizado ninguna fuente que se ajustara a los requerimientos generales de la estimación. Aunque a partir de 1891 la Dirección General de Agricultura, Industria y Comercio inauguraba una nueva estadística de precios de la producción para más de una treintena de productos agrarios que vieron la luz a través de tres publicaciones: Boletin Semanal de Estadistica y Mercados (1891-1902), Boletín Quincenal de Estadística, Mercados e Informaciones Agricolas (1903) y Boletin de Comercio e Información Agrícola y Estadística de Mercado (19041907), hemos desestimado su empleo porque, si bien para los granos y las carnes la información es sistemática en todos los mercados, no ocurre lo mismo con otros productos de consumo primario como son el arroz, las judías $y$ las patatas ${ }^{11}$. Nuestra estimación del índice del coste de la vida se ha basado en tres series de precios al por menor publicadas por Reher y Ballesteros (1993a), Pérez Sánchez (1996) y Pérez Castroviejo (1992) para las provincias de Madrid, Valladolid y Vizcaya. Hemos tomado de las series originales las cotizaciones del pan de trigo, los garbanzos, el arroz, el aceite, el vino, la carne de vaca y las patatas, y se ha procedido a aplicarles idénticas ponderaciones (ver tabla 1).

Finalmente, para el tercer subperíodo 1907-1924 el índice se sustenta sobre la información de precios que emana del Instituto de Reformas Sociales y que a partir de 1925 fue continuada por el Ministerio de Trabajo, Comercio e Industria hasta la Guerra Civil. A pesar de las deficiencias que se le señalan, esta información es de un valor inestimable por dos razones: el Insti-

10 J. Simpson (1995), p. 188.

11 El Grupo de Estudios de Historia Rural [GEHR] (1980, 1981a, 1981b) acometió la in. gente tarea de vaciar y publicar los estadillos de algunos precios contenidos en estos tres boletines, dando así continuidad a las series de Sánchez-Albornoz (1975). 
tuto recopiló precios al por menor y divulgó los datos a través de sus periódicos Boletines ${ }^{12}$.

Esta documentación no está exenta de problemas, especialmente para los años 1905-1908. En primer lugar, no existía ningún control que garantizara la actualización de las cotizaciones por los agentes responsables de recoger y remitir los precios. En segundo lugar, la periodicidad de la estadística entre 1905 y 1908 no es homogénea: se reunieron precios trimestrales, pero en cada período los precios corresponden a distintos grupos de provincias, de manera que la comparabilidad de los datos resulta muy arriesgada. A partir de 1909, esta deficiencia se subsanó y la periodicidad pasó a ser semestral para el conjunto de las 49 provincias. Algo similar ocurre con los contenidos: entre 1905 y 1908 la estadística de subsistencias reseña 15 productos, y a partir de 1909 se amplió a 42 incluyendo artículos no estrictamente alimenticios ${ }^{13}$. Aunque la fusión de la sección de subsistencias del Instituto de Reformas Sociales con un departamento de estadística del Ministerio de Trabajo por el Real Decreto 2/06/1924 supuso la reducción de las series a 17 productos, la periodicidad de los registros mejoró al ser publicada desde entonces con carácter mensual.

De esta fuente se han extraido los precios correspondientes a 15 artículos: pan de trigo, garbanzos, arroz, judías, patatas, aceite, vino, leche, carne de vaca, bacalao, huevos, azúcar y café como componentes de la alimentación y, además, se han considerado las cotizaciones del carbón y del jabón. Todos los precios, salvo los del carbón, se expresan en pesetas y unidades del sistema métrico decimal, por lo que no requieren ningún tratamiento de normalización. El carbón en 1907 y 1908 se expresa en pesetas por quintal y, entre 1909 a 1926, en pesetas por arroba, que hemos convertido a pesetas por kilogramo.

12 El mérito del Instituto de Reformas Sociales en su afán de elaborar informes monográficos sobre la problemática social de la España del momento está fuera de toda duda. En materia de precios, este es el juicio emitido por E. Riú (1917), pp. 5-51: «(...) este sería el organismo que más seriamente, con mayor preparación técnica y doctrinal podría acometer en España un servicio científico de precios de los artículos de primera necesidad, completándolo con el servicio de formación de presupuestos del coste de la vida del obrero por localidades». Asimismo hay que recordar que el trabajo clásico de S. Roldán (1972), pp. 5-66, se elaboró con los resúmenes de esta estadística y que, como el mismo indica, a pesar de las limitaciones es la que más confianza inspira.

13 Las inquietudes en la formación de la estadística y el proceso de elaboración se detallan en: Instituto de Reformas Sociales $(1916,1923)$; ver también, Boletin del Instituto de Reformas Sociales, tomo 57, Madrid, pp. 3-41. 
Existen, además de los citados, otros organismos que recopilaron precios tales como el Instituto Geográfico y Estadístico o las Cámaras de Comercio, pero la calidad de sus datos es netamente inferior a los de las publicaciones aquí señaladas ${ }^{14}$.

\subsection{Composición de los índices provinciales de la alimentación}

Del epígrafe anterior se desprende que aunque el material localizado no responde exactamente a los requerimientos iniciales, sí permite construir un índice del coste de la vida aceptable.

Nuestra estimación se sustenta sobre 276 series de precios, de las cuales 252 se refieren a artículos relativos a la alimentación. Esta característica no representa una grave deficiencia, ya que, como se recordará, la distribución del gasto familiar se mantuvo muy estable en la cronología de análisis y además en ella se atribuye a la alimentación más de dos tercios del gasto total ${ }^{15}$. Los productos incluidos bajo este epígrafe y la importancia atribuida a los mismos reflejan la composición interna del índice parcial de la alimentación (tabla 1). El principal componente de la dieta era el pan; su participación en el cáculo tiene una valuación decreciente en el tiempo $(0,50-0,30)$ en favor del realce que en el consumo fueron adquiriendo otros productos. Las legumbres $(0,15)$ y las patatas $(0,20)$ poseen en la ponderación un peso mayor que la carne $(0,10)$ y las grasas $(0,05)$ como consecuencia de las diferencias en el coste monetario de los bienes. La presencia del vino resulta ineludible por su aporte calórico aunque su importancia en el índice quizá haya sido subvalorada $(0,07)$.

\subsection{Participación de los precios de otros bienes}

Sería incorrecto no incluir en el índice los apartados relativos al vestuario, la vivienda y el combustible. Para cubrir el 30 por ciento restante del presupuesto familiar, hemos incorporado en el índice como indicador del

${ }_{14}$ Para una revisión crítica de las fuentes que suministran precios en el primer tercio del siglo XX remitimos a E. Riú (1917), pp. 5-51; E. André (1920), pp. 5-46; S. Roldán (1972), pp. 5-66; A. Ojeda Eiseley (1988), pp. 13-47. Para un período más amplio, ver J. Maluquer (1989), pp. 499-532.

${ }_{15}$ Sobre este aspecto, ver E. Ballesteros Doncel (1997), p. 12. 


\section{TABLA 1}

Composición del indice del coste de la alimentación en España, 1861-1936

\begin{tabular}{|c|c|c|c|}
\hline Artículos & Ponderación & Fuente & Localidades \\
\hline 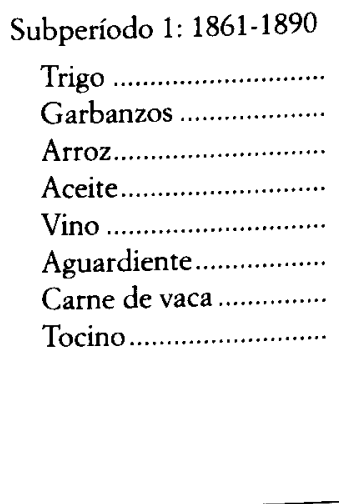 & $\begin{array}{l}0,500 \\
0,150 \\
0,050 \\
0,030 \\
0,070 \\
0,050 \\
0,050 \\
0,100\end{array}$ & La Gaceta de Madrid & $\begin{array}{l}\text { Alicante } \\
\text { Barcelona } \\
\text { Cáceres } \\
\text { La Coruña } \\
\text { Granada } \\
\text { Madrid } \\
\text { Navarra } \\
\text { Sevilla } \\
\text { Toledo } \\
\text { Valladolid } \\
\text { Vizcaya } \\
\text { Zaragoza }\end{array}$ \\
\hline $\begin{array}{r}\text { Subperíodo 2: } 1890-1907 \\
\text { Pan de trigo .................. } \\
\text { Garbanzos .................. } \\
\text { Arroz......................... } \\
\text { Aceite } \\
\text { Vino } \\
\text { Carne de vaca } \\
\text { Patatas }\end{array}$ & $\begin{array}{l}0,450 \\
0,100 \\
0,050 \\
0,030 \\
0,070 \\
0,100 \\
0,200\end{array}$ & $\begin{array}{l}\text { Reher y Ballesteros (1993b) } \\
\text { Pérez Sánchez (1996) } \\
\text { Pérez Castroviejo (1992) }\end{array}$ & $\begin{array}{l}\text { Madrid } \\
\text { Valladolid } \\
\text { Vizcaya }\end{array}$ \\
\hline
\end{tabular}

Subperíodo 3: 1907-1936

Pan de trigo

0,300

Garbanzos

0,100

Arroz.

0,025

Judías

0,025

Patatas

0,200

Aceite

0,050

Vino

0,050

Leche

0,030

Carne de vaca ................ $\quad 0,100$

Boletin del Instituto

Bacalao ........................ 0,050

Huevos...................... 0,030

Azúcar.......................... 0,020

Café * ......................... 0,020

Alicante

Barcelona

Cáceres

La Coruña

Granada

Madrid

Navarra

Sevilla

Toledo

Valladolid

Vizcaya

Zaragoza

* Este producto se incluye desde 1909. 
vestuario la serie de vestido y calzado elaborada por Reher y Ballesteros (1993a) y también el precio de la vivienda entre 1902 y 1931, estimado recientemente para Valladolid por Pérez Sánchez (1996). En lo que respecta al combustible, contamos con datos provinciales entre 1907 y 1926; el resto de la cronología se ha completado con los datos de Reher y Balleteros (1993a). Por último, el precio del jabón entre 1907 y 1936 proviene de las estadísticas elaboradas a partir de los Boletines del Instituto de Reformas Sociales y del Ministerio de Trabajo.

El resultado de combinar las distintas series de precios asociadas a las partidas de gasto queda reflejado en la tabla 2.

TABLA 2

Porcentajes de gasto asociados a los grupos de consumo

\begin{tabular}{lcccc}
\hline & \multicolumn{4}{c}{ Periodos } \\
\cline { 2 - 5 } Grupos de gasto & $1861-1901$ & $1902-1908$ & $1909-1931$ & $1932-1936$ \\
\hline Alimentación ............... & 75,0 & 70,0 & 70,0 & 70,0 \\
Vestuario .................... & 12,5 & 10,0 & 10,0 & 15,0 \\
Vivienda .................. & - & 10,0 & 10,0 & - \\
Otros: & 12,5 & 10,0 & 5,0 & 10,0 \\
Combustible .............. & - & - & 5,0 & 5,0 \\
Jabón....................................................... & 100,0 & 100,0 & 100,0 & 100,0 \\
\hline
\end{tabular}

\subsection{Estimación y alcance de índice del coste de la vida}

El proceso seguido para culminar el objetivo inicial puede describirse de la siguiente manera. Para cada subperiodo se construyen números índices con las series anuales de precios provinciales, a las que se aplican las ponderaciones descritas en la tabla 1 , los cálculos se resuelven mediante una fórmula de tipo Laspeyres, obteniendo los índices provinciales del coste de la alimentación. Los resultados se pueden consultar en las tablas 5, 6 y 7 del apéndice.

Sobre estos indices provinciales de la alimentación se establece la media, alcanzándose el promedio del país. Así se llega a disponer de tres índices, uno para cada subperíodo, que son enlazados al convertir a base común las series de distinta cronología. Técnicamente esta forma de encadenamiento 
presenta el inconveniente de que hace iguales series de composición diferente. Sin embargo, este procedimiento se ha utilizado en España y en otros países en aquellas épocas en que aún no se halla configurada la moderna estadística socio-económica. La necesidad de conocer la evolución histórica de los precios prima sobre el rigor ciéntifico de neutralizar los cambios en los patrones de consumo ${ }^{16}$.

Finalmente, a la serie parcial de la alimentación se le incorporan los índices de otros bienes: vestuario, vivienda combustible y jabón. Se calcula de nuevo un índice siguiendo la fórmula Laspyres con los pesos descritos en la tabla 2 y se llega al índice del coste de la vida, cuyos resultados se recogen en la tabla 8 del apéndice estadístico.

La estimación llevada a cabo en este ejercicio supone un avance en el conocimiento de los precios en España, puesto que incorpora la recreación de un conjunto de series de precios relativas a doce provincias de la geografía peninsular para los períodos 1861-1890 y 1907-1936. La principal contribución de este trabajo está en relación con la recopilación de cotizaciones de bienes relativos a la alimentación, mientras que la cobertura del índice sobre el resto de los bienes puede valorarse como indicativa, especialmente para la vivienda y el vestuario.

Las limitaciones más importantes de nuestro cálculo tienen dos dimensiones. En primer lugar, con respecto a la naturaleza de las cotizaciones, para el primer subperíodo (1861-1890) disponemos de precios de producción, en contraste con el resto de la cronología que contamos con precios al por menor. En segundo lugar, para el segundo subperíodo (1891-1907), el estado de las fuentes nos ha impedido recrear las valiosas series provinciales y así hemos utilizado los datos locales disponibles para Madrid, Valladolid y Vizcaya. Este es, a nuestro juicio, el problema más importante que presenta el cálculo, pero, pese a ello, la nueva estimación del coste de la vida mejora el conocimiento de los precios entre la segunda mitad del siglo XIX y el primer tercio del siglo XX.

\section{RESULTADOS}

\subsection{Comportamiento de la serie}

Los resultados obtenidos se han plasmado en el gráfico 1, donde lo más sobresaliente, a primera vista, es el dualismo que en el largo plazo muestra la

16 Sobre este asunto, ver Instituto Nacional de Estadística (1979), pp. 29-32. 


\section{GRÁFICO 1}

Indice del coste de la vida en España, 1861-1936 (base $1913=100$ )

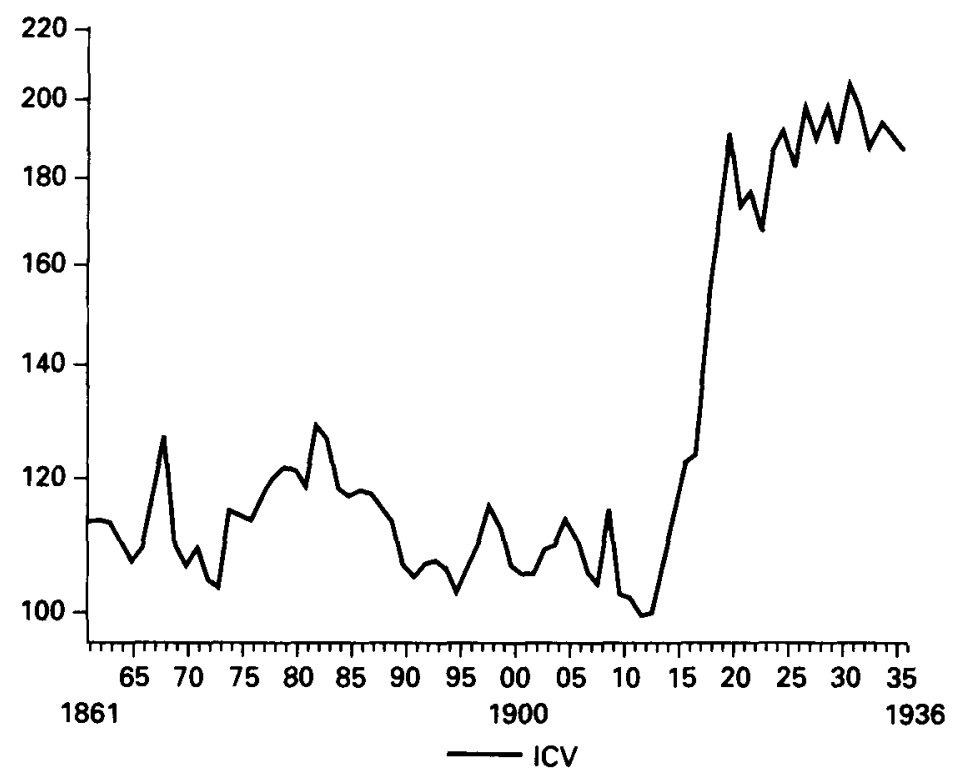

FUENTE: Ver texto y tablas 1 y 8 .

serie. La relativa estabilidad del novecientos se ve sacudida por el proceso inflacionario ocurrido entre 1914 y 1920, conclusión que no modifica lo que ya sabíamos.

Ahora bien, el alcance de las crisis de subsistencias de la segunda mitad del siglo XIX ha sido medido, hasta el momento, considerando el movimiento en el precio de los cereales. Según Sánchez-Albornoz, el incremento en la cotización del trigo fue del 56 por ciento en 1868 , del 27 por ciento en 1879 y del 34 por ciento en $1882^{17}$. La importancia del trigo en el consumo global de la población no justifica que se pueda establecer una identidad entre crisis de cereales y crisis de subsistencias. Si medimos el aumento, para esos mismos años, sobre nuestro índice del coste de la alimentación (aislando las variaciones del vestuario y el combustible), las cifras se atenúan no-

17 N. Sánchez-Albornoz (1975), pp. 34-35. 
tablemente. Entre 1866 y 1868 el encarecimiento no superó el 26 por ciento; mientras que entre 1877-1879 y 1881-1882 el índice de precios subió un $10 \mathrm{y}$ un 11 por ciento, respectivamente.

Esta reducción significativa sobre el efecto de las crisis de subsistencias confirma su existencia y, al mismo tiempo, sugiere una lectura renovada de su alcance; pero sobre todo invita a proyectar análisis menos simplificados sobre la relación de variables económicas y sociales. En este sentido, también nuestro estudio podría ser severamente juzgado si echáramos en el olvido la importancia del autoconsumo concretada en la tenencia de tierra o en el disfrute de una pequeña parcela, la cría de animales de granjería, el acceso a la pesca fluvial y marítima, la caza, la recogida de frutos, etc., en un contexto de preeminencia del campesinado. Además, la propia experiencia indica que la carestía o encarecimiento de un producto forzará a sustituirlo o complementarlo con otros bienes, y esta estrategia no ha sido controlada en el análisis. El estudio de las crisis de subsistencia pasa por un conocimiento más profundo de la estructura social y de las relaciones que en ella operan. La incidencia de ciertos factores puede suponer que la población mantiene un cierto bienestar aun cuando los precios sugieran una pérdida del mismo.

Volviendo a la trayectoria que marca el índice del coste de la vida: la deflación de los años ochenta y noventa del siglo pasado se explica tanto por el contexto internacional depresivo de la economía como por el progresivo efecto en la depreciación de la peseta. Las implicaciones del conflicto hispano-americano sobre los precios, a juzgar por el comportamiento del índice, no resultaron significativas, con un aumento del 10 por ciento en el trienio 1897-1899.

El cambio de siglo no alteró la dinámica, pues la moderada sucesión de alzas y bajas, nunca superiores al 10 por ciento, se compensan entre sí y dibujan una tendencia plana de inapreciable pendiente.

El punto de inflexión en el comportamiento de la serie se localiza en el año 1914. La vertiginosa inflación registrada en los precios estuvo determinada en buena medida por los efectos que sobre nuestra economía desencadenó la Gran Guerra. Pero no se puede olvidar que en ese momento España vivía un alto grado de conflictividad social, como lo demuestran las huelgas generales de los años 1916 y 1917. El valor del índice no sólo se dobló entre 1914 y 1920, sino que además mantuvo ese nivel hasta el final de la serie. El barómetro de los precios refleja fundamentalmente la dinámica económica pero también es sensible a los hechos políticos y sociales; resolver el peso de cada factor sobre su evolución en el tiempo forma parte de una nueva investigación. 


\subsection{Comparación con otras series}

La comparación entre el nuevo índice del coste de la vida y las series de Sardá para la ciudad de Barcelona, y de Reher y Ballesteros para Madrid, se ha establecido eliminando la tendencia a partir de las primeras diferencias, obteniendo los siguientes valores de correlación positiva (tabla 3 ).

\section{TABLA 3}

Coeficientes de correlación entre los indices del coste de la vida en España, Madrid y Barcelona

\begin{tabular}{|c|c|c|c|}
\hline & $I C V$ & REHER & SARDÁ \\
\hline 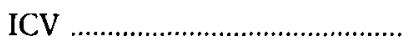 & $1,000^{*}$ & & \\
\hline REHER & $0,562^{\star}$ & $1,000^{*}$ & \\
\hline SARDÁ & $0,458^{*}$ & $0,499^{*}$ & $1,000^{\star}$ \\
\hline
\end{tabular}

* Nivel de significación 0,01 .

Con la serie de Sardá el coeficiente alcanza un modesto valor, 0,458 , mientras que con la de Madrid se eleva a 0,562, y entre ellas, el coeficiente es de 0,499. En nuestra opinión, la discreta intensidad en la relación observada entre los tres índices responde a dos factores.

En primer lugar, la naturaleza de los precios condiciona la evolución de las series. Hay que recordar que los datos de Sardá son en su mayoría de importación, el índice de Reher y Ballesteros se nutre de cotizaciones al por menor y la nueva serie, como ya hemos explicado, posee un carácter mixto. Obviamente, en el largo plazo los principales impulsos de nuestra economía se reproducen en las tres series, aunque sin duda a corto y medio plazo existen divergencias significativas.

El gráfico 2 muestra que la distancia entre las series se establece básicamente en dos momentos. Por un lado, la depresión de los años ochenta y noventa es más acusada en el índice de Sardá que en los otros dos y, por otro lado, la inflación iniciada en 1914 alcanza mayor virulencia también en la serie relativa a la ciudad de Barcelona, que además es la única de las tres que muestra un considerable descenso posterior al conflicto europeo. Como se comprobará más adelante, las características que muestra el índice de Sardá son más afines a la trayectoria seguida por Inglaterra, Francia e Italia que a nuestra estimación del coste de la vida en España. 


\section{GRÁFICO 2}

Indices de precios en España, Madrid y Barcelona, 1861-1936

(base $1913=100)$

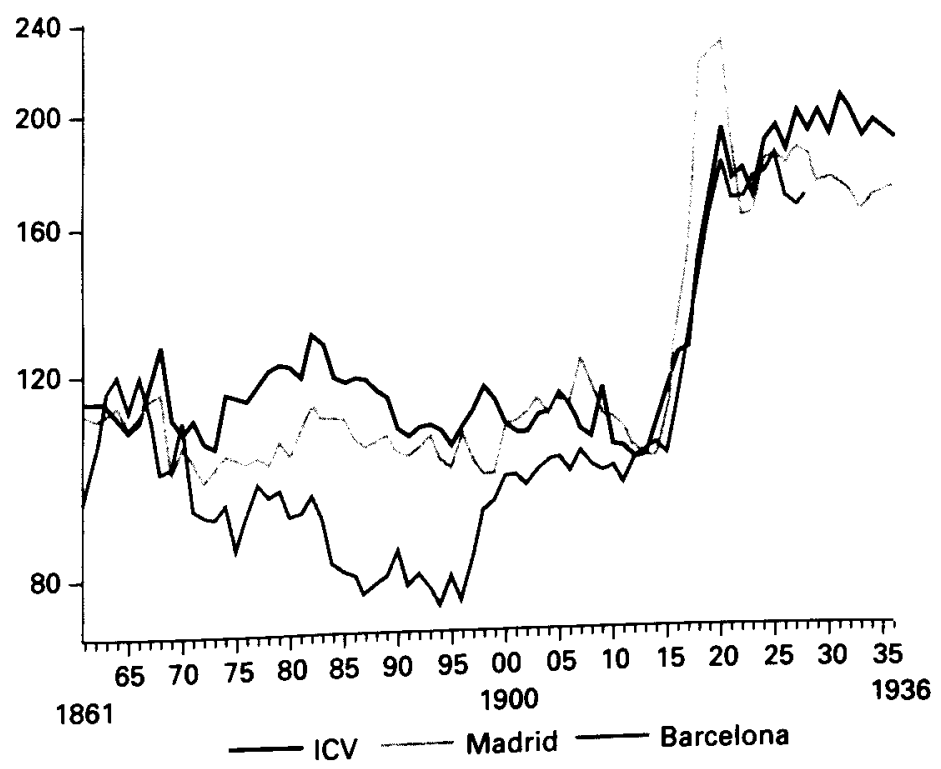

Fuentes: Madrid, Reher y Ballesteros (1993a), pp. 135-136; Barcelona, Sardá (1948), pp. 302-305.

El segundo factor de desigualdad es el grado de integración del mercado nacional. Aunque nuestra estadística no permite resolver esta cuestión sí puede explorarla. Se han calculado dos matrices de correlación para los subperíodos en los que poseemos datos en las doce provincias (tablas 4 y 5) y los resultados indican una gran armonía.

En la tabla 4 los valores de correlación obtenidos para 1861-1890 sugieren que los índices de precios provinciales se encuentran en sintonía. Según estos datos, España poseía en la segunda mitad del siglo XIX un merca. do interior sólido e interdependiente. La Coruña es la unidad más divergente de todas las consideradas, seguida de Sevilla, Vizcaya y Barcelona. Estas provincias tienen en común que son economías abiertas al mercado marítimo, lo que se traduce en una ventaja comercial ante eventuales adversidades. 


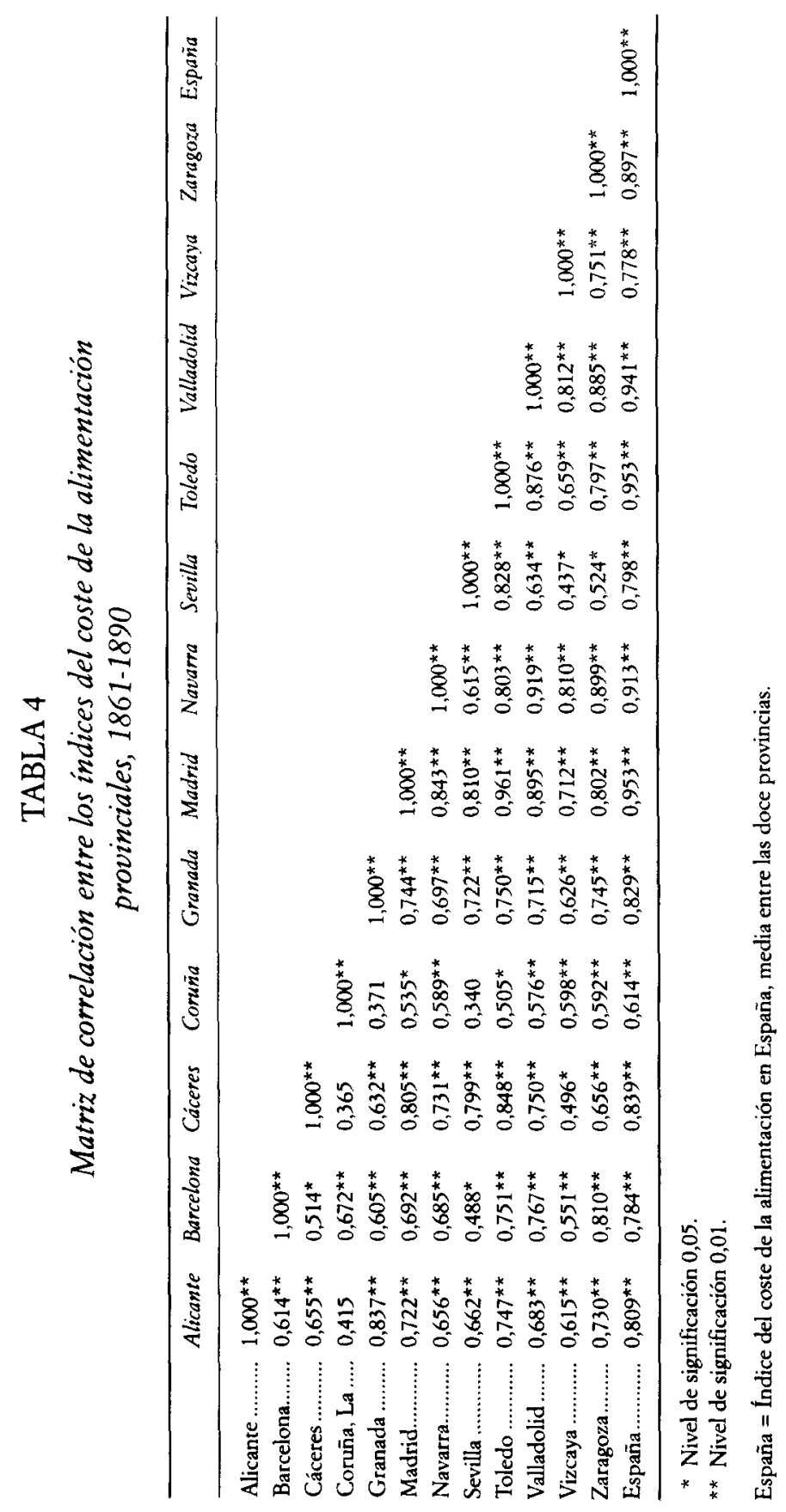




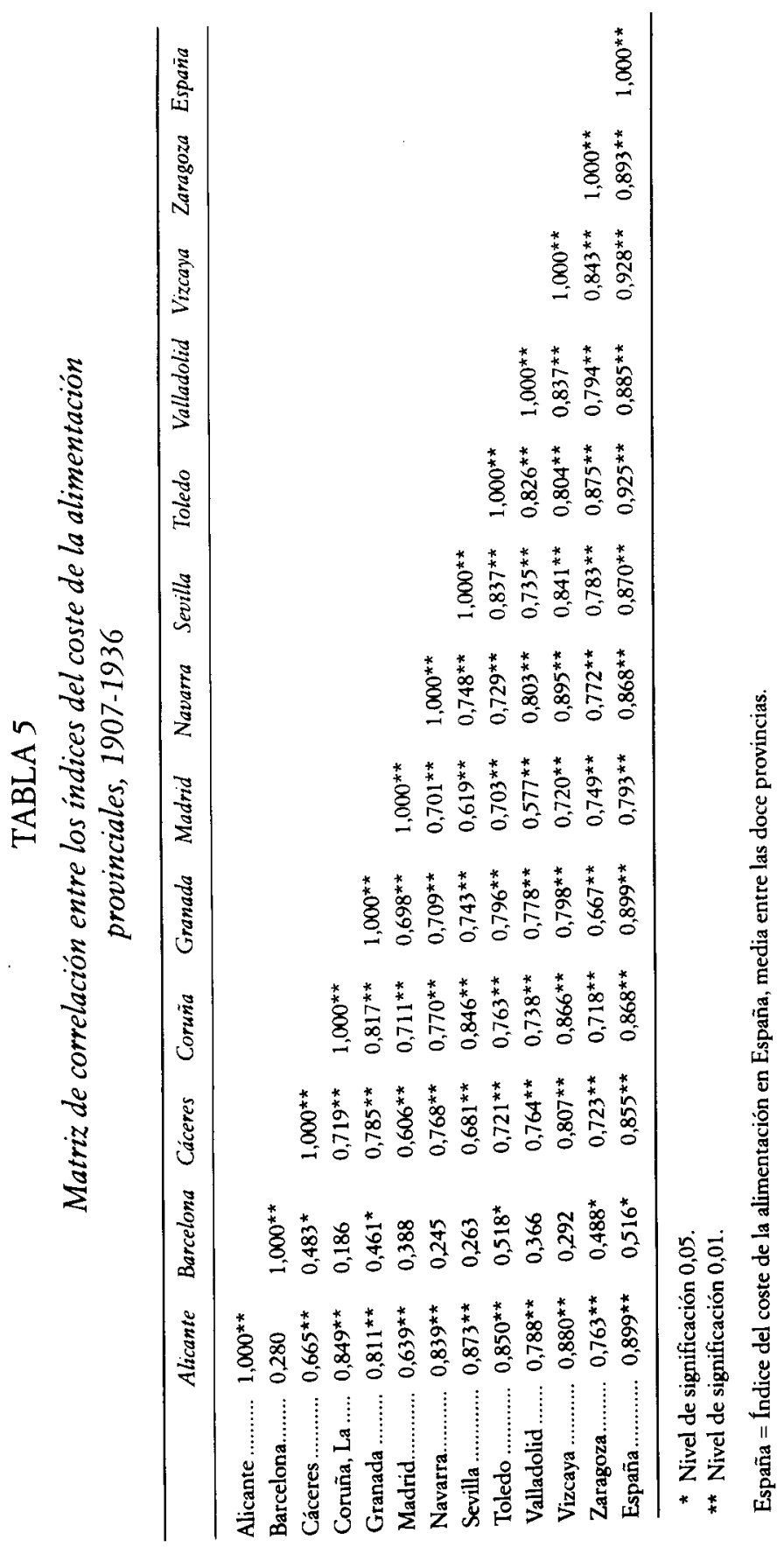


Para el período 1907-1936, como cabía esperar, los coeficientes son aún mayores (tabla 5). Aunque en este caso lo significativo es que la provincia de Barcelona se desvía notablemente del patrón común marcado por las restantes once unidades. Esta situación quizá indique que la economía barcelonesa pudiera estar más integrada en el mercado exterior que en el mercado nacional, pero esta afirmación, por el momento, sólo representa una hipótesis.

\subsection{Comparación con otros países europeos}

La comparación del índice del coste de la vida en España se ha hecho con cuatro países, Francia y Gran Bretaña como núcleos de rápido desarrollo, y Portugal e Italia como áreas menos aventajadas. En el gráfico 3 se puede observar la evolución de los índices de precios en España, Portugal e

\section{GRÁFICO 3}

Indices de precios en España, Portugal e Italia, 1861-1936

(base $1913=100$ )

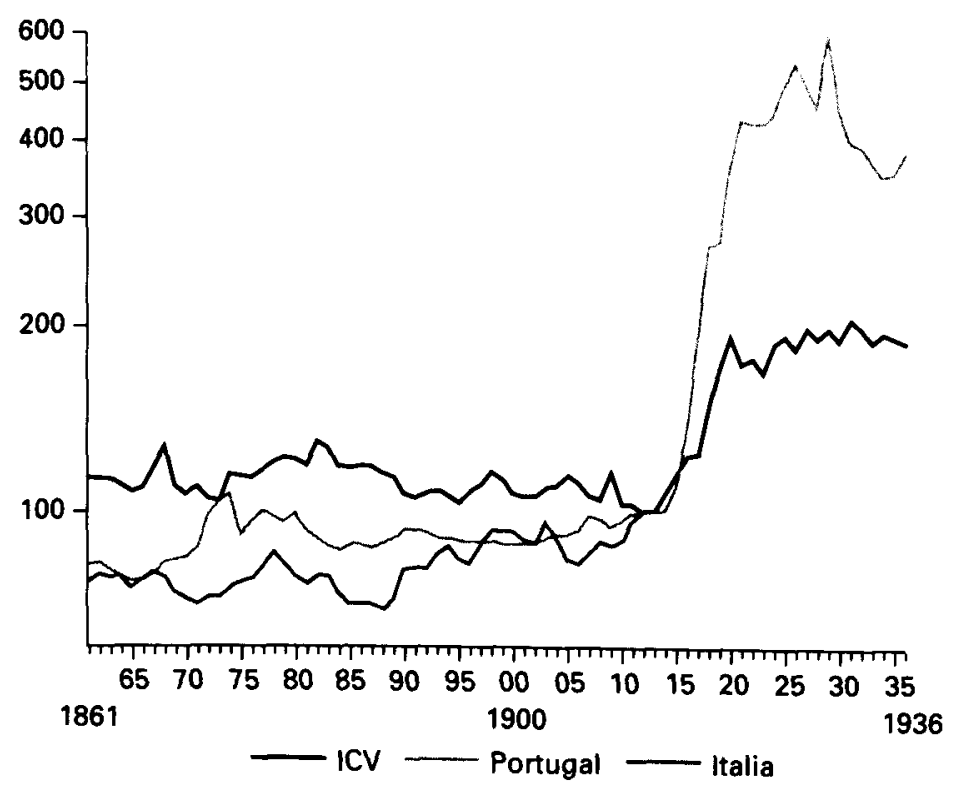

FuENTES: Portugal, Justino (1989), pp. 14-16; Italia, Mitchell (1975), pp. 742-747. 


\section{GRÁFICO 4}

Indices de precios en España, Inglaterra y Francia, 1861-1936

(base 1913 = 100)

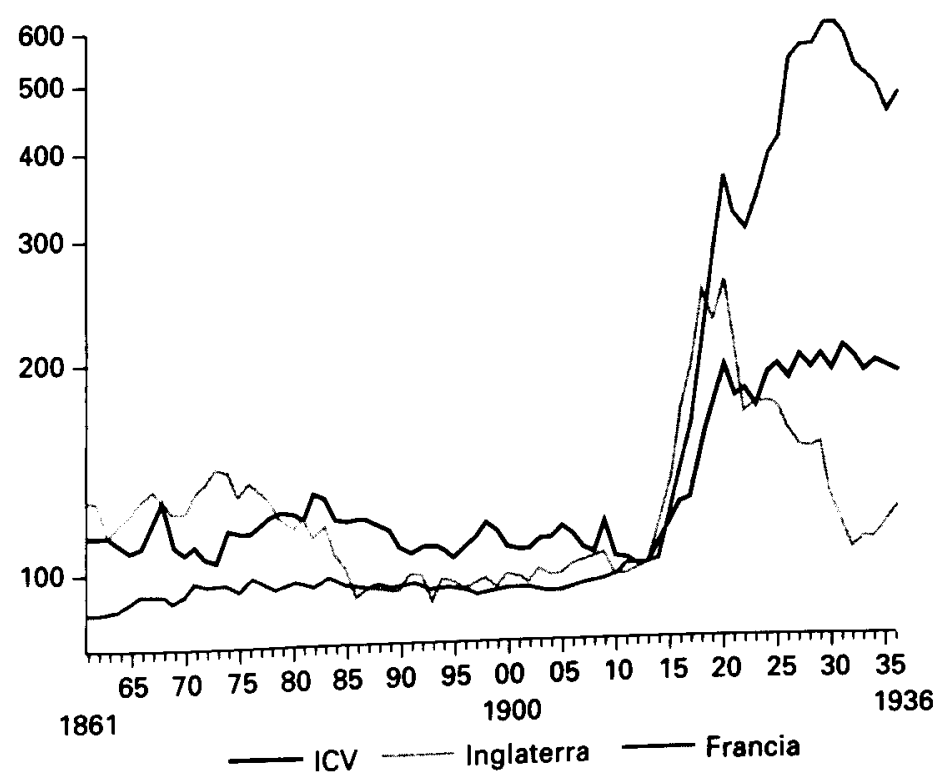

FuENTES: Inglaterra, Phelps Brown y Hopkins (1956), pp. 299-302; Francia, Mitchell (1975), pp. $742-747$.

Italia. En el largo plazo los tres indicadores muestran el contraste entre la relativa estabilidad del novecientos y la inflación en torno a los años de la Primera Guerra Mundial. La intensidad en los años de depresión e inflación resulta mayor en Italia que en España.

Por último, la experiencia de Inglaterra y Francia frente a España resulta positiva en el medio y largo plazo (gráfico 4). El proceso de deflación en ambos países es más acusado que en España; asimismo, la inflación del período bélico tuvo un mayor efecto para Francia e Inglaterra.

\section{CONCLUSIONES}

El material generado en este artículo acrece el interés por el estudio de los precios en España. Somos conscientes que la estimación del coste de la 
vida realizada se presta a mejoras sustanciales, especialmente en el período que transcurre entre 1891 y 1906 cuando el índice recoge los precios en tan sólo tres provincias. A pesar de ello, las nuevas series presentadas pueden contribuir eficazmente al desarrollo de varios debates. A la luz de los nuevos resultados, los efectos de las crisis de subsistencias invitan a proyectar análisis más sofisticados sobre la relación de variables sociales y económicas. Queda por determinar, entre otras cosas, la elasticidad de la demanda en los productos considerados de consumo primario. Por otra parte, los datos sugieren que la articulación del mercado interior podría haber alcanzado en la segunda mitad del novecientos un alto grado de madurez, pero la confirmación de esta hipótesis exige tomar en cuenta nuevos elementos.

Tanto la estimación del índice del coste de la vida en España como las series de precios provinciales que lo conforman pueden enriquecer otros trabajos de muy diversa índole. Sea como fuere, el conocimiento de los precios sigue siendo asunto muy atractivo para los estudiosos. Esta investigación así lo confirma.

\section{BIBLIOGRAFIA}

\section{Publicaciones periódicas}

BOLETIN DEL INSTITUTO DE REFORMAS SOCIALES (1906-1924).

Boletin Oficial del MinisTerio de Trabajo (1924-1936).

LA GACETA DE MADRID (1861-1890).

\section{Libros y artículos}

ANDRÉ, E. L. (1920): «Subsistencias y precios», Revista Nacional de Economía, año $\mathrm{V}, \mathrm{n}^{\circ}{ }^{2} 23$, Madrid, pp. 5-46.

BALLESTEROS DONCEL, E. (1997): «El presupuesto familiar como indicador de los niveles de vidam, III Seminario sobre economias y estrategias familiares. Seminario sobre mercado de trabajo y migraciones, movilidad social, trabajo femenino y asistencia a la tercera edad, Universitat Pompeu Fabra, 3-5/03/1997.

DeAnE, P., y COLE, W. A. (1962): British Economic Growth, 1688-1959, Cambridge, Cambridge University Press.

DIRECCIÓN GENERAL DEL TRABAJO (1931): Estadistica de salarios y jornadas de trabajo, 1914-1930, Madrid.

FEINSTEIN, CH. (1995): «Nominal wages, cost of living and real wages in the UK», en P. Scholliers y V. Zagmani (eds.) (1995): Labour's Reward, Real Wages and economic change in 19th and 20th century Europe, Ed. Edward Elgar, pp. 3-35. 
FELIÚ, G. (1991): Precios y salarios en la Cataluña Moderna (2 vols.), Serie de Historia Económica, $n .^{\text {os }} 21$ y 22 , Madrid, Servicio de Estudios del Banco de España.

FLINN, M. W. (1974): «Trends in Real Wages, 1750-1850», The Economic History Review, XXVII, 3, pp. 395-413.

GARCIA SANZ, A. (1980): «Jornales agrícolas y presupuesto familiar campesino a mediados del siglo XIX», Anales de Cunef, 1979-80, pp. 50-71.

GRUPO DE ESTUDIOS DE HISTORIA RURAL (1980): Los precios del trigo y la cebada en España, 1891-1907, Serie de Historia Económica, n. ${ }^{\circ}$ 1, Madrid, Servicio de Estudios del Banco de España.

- (1981a): Los precios del aceite de oliva en España, 1891-1916, Serie de Historia Económica, n. ${ }^{\circ} 4$, Madrid, Servicio de Estudios del Banco de España.

- (1981b): El vino, 1874-1907, dificultades para reconstruir la serie de sus cotizaciones, Serie de Historia Económica, n. ${ }^{\circ}$ 6, Madrid, Servicio de Estudios del Banco de España.

HAMILTON, E. (1975): El tesoro americano y la revolución de los precios en España, 1501-1650, Barcelona, Ed. Ariel, 1983 (2." edición).

INSTITUTO DE REFORMAS SOCIALES (1916): Coste de la vida del obrero. Estadística de los precios de los artículos de primera necesidad en toda España, 1909-1915, Madrid.

(1923): «Movimiento de los precios al por menor en España. Durante la guerra y la postguerra, 1914-1922», Boletin del Instituto de Reformas Sociales, 57, pp. 3-41.

INSTITUTO GEOGRÁFICO Y ESTADISTICO (1903): Estadística de la emigración e inmigración de España en el quinquenio 1896-1900 (bienio 1896/97).

- (1916 y 1931): Anuario Estadístico de España, Madrid.

InSTITUTO NACIONAL DE ESTADISTICA (1979): Indice de precios de consumo. Monografia técnica, Madrid, Ed. INE.

Justino, D. (1989): A formaçáo do espaço económico nacional. Portugal, 1810-1913, Lisboa.

KONDO, A. (1990): La agricultura española en el siglo XIX, Madrid, Ministerio de Agricultura, Pesca y Alimentación, Ed. Nerea.

LABROUSSE, E. (1962): Fluctuaciones económicas e Historia Social, Madrid, Ed. Tecnos, 1980 (2." edición).

Madrazo MadRazO, S. (1984): El sistema de comunicaciones en España, Madrid, Ed. Colegio de Ingenieros de Caminos Canales y Puertos y Turner.

MALUQUeR, J. (1989): «Precios, salarios y beneficios. La distribución funcional de la renta», en Estadísticas Históricas de España, siglos XIX-XX, Madrid, Ed. Fundación Banco Exterior.

MARTÍN ACEÑA, P. (1992): «Los precios en Europa durante los siglos XVI y XVII. Un estudio comparativo», Revista de Historia Económica, X, 3, pp. 359-395.

MITCHELL, B. R. (1975): European Historical Statistics, 1750-1970, Londres.

OJEDA EISELEY, A. (1988): Indices de precios en España en el periodo 1913-1987, Serie de Historia Económica, n. ${ }^{\circ} 17$, Madrid, Servicio de Estudios del Banco de España.

Pérez Castroviejo, P. (1992): Clase obrera y niveles de vida en las primeras fases de la industrialización vizcaina, Madrid, Ed. Ministerio de Trabajo y Seguridad Social. 
PÉREZ SÁNCHEZ, G. (1996): Ser trabajador: vida y respuesta obrera. Valladolid, 1875 1931, Valladolid, Ed. Secretariado de Publicaciones e Intercambio Científico de la Universidad de Valladolid.

Phelps Brown, E. H., y Hopkins, S. V. (1956): «Seven Centuries of the Prices of Consumables, Compared with Builders' Wages-Rates», Economica, XXIII, 92, pp. 296-314.

REHER, D., y BALlesteros, E. (1993a): «Precios y salarios en Castilla la Nueva. La construcción de un índice de salarios reales, 1501-1991», Revista de Historia Económica, XI, 1, pp. 101-151.

- (1993b): Indicadores de la dinámica económica en Madrid. Precios y salarios, 1800-1991, Documento de Trabajo n. ${ }^{\circ}$, Madrid, Instituto de Demografía (CSIC).

REHER, D., y CAMPS, E. (1991): «Las economías familiares dentro de un contexto histórico comparado», Revista Española de Investigaciones Sociológicas, 55, pp. 65-91.

RuU, E. (1917): «El problema de las subsistencias», Revista Nacional de Economía, II, 5, Madrid, pp. 5-51.

ROLDÁN, S. (1972): «Precios, salarios y conflictos. El proceso inflacionista entre 1914 y 1920», Revista de Trabajo, 37, pp. 5-66.

SARDÁ, J. (1948): La política monetaria y las fluctuaciones de la economia espabila en el siglo XIX, Madrid, Consejo Superior de Investigaciones Científicas.

SÁNCHEZ-ALBORNOZ, N. (1968): España hace un siglo, una economia dual, Madrid, Alianza Universidad, 1988 (3.a edición).

(1975): Los precios agricolas durante la segunda mitad del siglo XIX. Trigo y cebada, Madrid, Servicio de Estudios del Banco de España.

— y CARNERO ARBAT, T. (1981): Los precios agricolas durante la segunda mitad del siglo XIX. Aceite y vino, Madrid, Servicio de Estudios del Banco de España.

SIMPSON, J. (1989a): «La producción agraria y el consumo español en el siglo XIX», Revista de Historia Económica, VII, 2, pp. 355-388.

_- (1995): «Real wages and labour mobility in Spain, 1860-1936», en P. Scholliers y V. Zamagni (eds.) (1995): Labour's Reward. Real wages and economic change in 19th and 20th century Europe, Hants, Edward Elgar, 1995, pp. 182-199.

Williamson, J. G. (1989): Capitalismo y desigualdad económica en Gran Bretaña, Madrid, Ed. Ministerio de Trabajo y Seguridad Social.

\section{APÉNDICE}

NOTA: Las normas establecidas por la Revista de Historia Económica para la publicación de artículos limitan la extensión de los originales, lo que nos ha impedido incluir en el trabajo las series anuales de precios. La autora advierte que el material de investigación está a disposición de los interesados y que podrá solicitarse a través de la Secretaría de Redacción de la Revista. 
TABLA 1

Precios del trigo, jornales diarios (en pesetas) y nümeros indices en 1860

\begin{tabular}{|c|c|c|c|c|c|c|c|}
\hline & \multirow{2}{*}{ 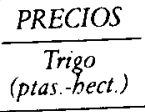 } & \multicolumn{3}{|c|}{ JORNALES } & \multicolumn{3}{|c|}{ INDICES (base, España $=100)$} \\
\hline & & $\begin{array}{c}A 1860 \\
(1)\end{array}$ & $\begin{array}{c}\text { U1860 } \\
(2)\end{array}$ & $\begin{array}{c}\text { Media } \\
(3)\end{array}$ & $\begin{array}{c}\text { Precios } \\
\text { (4) }\end{array}$ & $\begin{array}{c}\text { Jornales } \\
(5)\end{array}$ & $\begin{array}{c}\text { Salario real } \\
(6)\end{array}$ \\
\hline Álava ....... & 19,50 & 1,00 & 2,13 & 1,56 & 92,71 & 117,82 & 127,08 \\
\hline Albacete. & 21,91 & - & 1,45 & 1,45 & 104,17 & 109,16 & 104,79 \\
\hline Alicante... & 26.62 & 1,13 & 1,58 & 1,35 & 126,56 & 102,01 & 80,60 \\
\hline Almería ..................... & 27,23 & 1,25 & 1.50 & 1,38 & 129,46 & 103,51 & 79,96 \\
\hline Ávila......... & 16,82 & 1,13 & 1,50 & 1,31 & 79,97 & 99.00 & 123,80 \\
\hline Badajoz ........ & 20.72 & 1,00 & 1,63 & 1,31 & 98,51 & 99,00 & 100,49 \\
\hline Barcelona .... & 26,02 & 1,75 & 2,05 & 1,90 & 123,71 & 143,04 & 115,63 \\
\hline Burgos ........... & 17,49 & - & 1,55 & 1,55 & 83,15 & 116,69 & 140,33 \\
\hline Cáceres..... & 18,67 & 0,88 & 1,25 & 1,06 & 88.76 & 80,18 & 90,33 \\
\hline Cádiz ........ & 25,88 & - & 1,90 & 1,90 & 123,04 & 143,04 & 116,25 \\
\hline Castellón.. & 21,49 & 1,00 & 1,50 & 1,25 & 102,17 & 94,10 & 92,10 \\
\hline C. Real ........ & 20,05 & 0,78 & 1,75 & 1,26 & 95,33 & 95,23 & 99,90 \\
\hline Córdoba... & 21,01 & 1,00 & 1,45 & 1,22 & 99,89 & 92,22 & 92,32 \\
\hline Coruña, La... & 22,66 & - & 1,28 & 1,28 & 107,73 & 96,36 & 89,44 \\
\hline Cuenca ........... & 17,21 & 1,25 & 1,53 & 1,39 & 81,82 & 104,64 & 127,89 \\
\hline Gerona............ & 23,27 & 1,06 & 1,60 & 1,33 & 110,63 & 100,13 & 90,50 \\
\hline Granada... & 25,54 & 1,00 & 1,50 & 1,25 & 121,43 & 94,10 & 77,50 \\
\hline Guadalajara & 16,22 & - & 1,55 & 1,55 & 77,12 & 116,69 & 151,32 \\
\hline Guipúzcoa.. & 23.53 & - & 1,93 & 1,93 & 111,87 & 145,30 & 129,88 \\
\hline Huelva ....... & 26,05 & 1,25 & 1.53 & 1,39 & 123,85 & 104,64 & 84,49 \\
\hline Huesca ................ & 21,30 & 1,13 & 1,75 & 1,44 & 101,27 & 108,41 & 107,05 \\
\hline 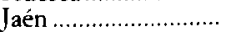 & 20,29 & 1,13 & 1,60 & 1,36 & 96,47 & 102,76 & 106,53 \\
\hline 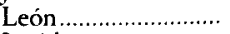 & 16,57 & 0,63 & 1,30 & 0,96 & 78,78 & 72,65 & 92,22 \\
\hline Lérida... & 25,46 & 1,25 & 1,75 & 1,50 & 121,05 & 112,93 & 93,29 \\
\hline Logroño...... & 17,93 & 0,94 & 1,55 & 1,24 & 85,25 & 93,73 & 109,95 \\
\hline Lugo........................... & 17,98 & 1,00 & 1.08 & 1,04 & 85,48 & 78,29 & 91,59 \\
\hline Madrid ........ & 18,88 & 1,25 & 1,63 & 1,44 & 89,76 & 108,41 & 120,77 \\
\hline Málaga ........ & 25,98 & 1,25 & 1,63 & 1,44 & 123,52 & 108,41 & 87,77 \\
\hline Murcia. & 28,06 & 1,00 & 1,68 & 1,34 & 133,41 & 100,88 & 75,62 \\
\hline 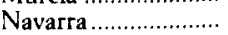 & 19,19 & 1.50 & 1,58 & 1,54 & 91,24 & 115,94 & 127,07 \\
\hline 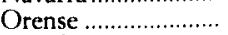 & 20,67 & 0,88 & 1,13 & 1,00 & 98,27 & 75,66 & 76,99 \\
\hline Oviedo & 22,91 & 1,00 & 1,43 & 1,21 & 108,92 & 91,47 & 83,98 \\
\hline Palencia....... & 16,92 & 0,95 & 1,58 & 1,26 & 80,44 & 95,23 & 118,38 \\
\hline Pontevedra................. & 25,53 & 0,63 & 1,13 & 0,88 & 121,38 & 66,25 & 54,58 \\
\hline Salamanca ................. & 15,03 & 0,88 & 1,50 & 1,19 & 71,46 & 89,59 & 125,37 \\
\hline Santander................. & 20,91 & - & 1,98 & 1,98 & 99,41 & 149,06 & 149,94 \\
\hline 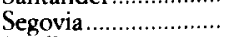 & 15,35 & - & 1,75 & 1,75 & 72,98 & 131,75 & 180,52 \\
\hline Sevilla...... & 22,55 & 1,25 & 1,75 & 1.50 & 107,21 & 112,93 & 105,33 \\
\hline Soria ................. & 16,29 & 1,00 & 1,25 & 1,13 & 77,45 & 84,69 & 109,35 \\
\hline Tarragona... & 26,78 & 1,13 & 1,73 & 1,43 & 127,32 & 107,66 & 84,55 \\
\hline Teruel & 19,48 & 1,13 & 1,48 & 1,30 & 92,62 & 98,25 & 106,08 \\
\hline Toledo & 18,50 & 1,00 & 1,50 & 1,25 & 87,96 & 94,10 & 106,99 \\
\hline Valencia. & 23,80 & 1,00 & 1,63 & 1.31 & 113,15 & 99,00 & 87,49 \\
\hline Valladolid.... & 17,56 & 0,88 & 1,50 & 1,19 & 83,49 & 89,59 & 107,31 \\
\hline Vizcaya....................... & 22,02 & 1,25 & 2,25 & 1,75 & 104,69 & 131,75 & 125,84 \\
\hline 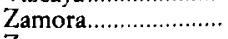 & 16,36 & - & 1,48 & 1,48 & 77,78 & 111,42 & 143,25 \\
\hline Zaragoza ................... & 18,37 & 1,25 & 1,63 & 1,44 & 87,34 & 108,41 & 124,12 \\
\hline Espa & 21,033 & 1,073 & 1,583 & 1,328 & 100,00 & $\begin{array}{l}100,00 \\
105,64\end{array}$ & $\begin{array}{l}100,00 \\
103,93\end{array}$ \\
\hline & 21,380 & 1,190 & 1,620 & 1,400 & 101,65 & 105,64 & 103,93 \\
\hline
\end{tabular}

Muestra ................. 21,380. en la construcción de caminos; (3): Media entre (1): Jornal medio de peones agrícolas; (2): Jornal de peones en la construcción de cas jornales y media de Es(1) y (2); (4): Cociente precio

FuENTES: Precio del trigo, N. Sánchez-Albomoz (1975), pp. 162-165; Jornales agrícolas, A. García Sanz (1980), p. 63; Jornales no agrícolas, S. Madrazo (1984), p. 208. 
TABLA 2

Precios del trigo, jornales diarios (en pesetas) y números indices en 1896

\begin{tabular}{|c|c|c|c|c|c|c|c|}
\hline & \multirow{2}{*}{ 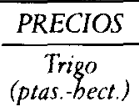 } & \multicolumn{3}{|c|}{ JORNALES } & \multicolumn{3}{|c|}{ INDICES (base, España $=100)$} \\
\hline & & $\begin{array}{c}A 1896 \\
\text { (1) }\end{array}$ & $\begin{array}{l}U 1896 \\
(2)\end{array}$ & $\begin{array}{c}\text { Media } \\
\text { (3) }\end{array}$ & $\begin{array}{l}\text { Precios } \\
\text { (4) }\end{array}$ & $\begin{array}{c}\text { Jornales } \\
(5)\end{array}$ & $\begin{array}{c}\text { Salario real } \\
\text { (6) }\end{array}$ \\
\hline Alava & 19,66 & 1,54 & 3,25 & 2,39 & 93,89 & 99,93 & 106,43 \\
\hline Albacete.. & 21,19 & 1,60 & 2,75 & 2,17 & 101,20 & 90,75 & 89,68 \\
\hline Alicante... & 22,18 & $\begin{array}{l}1,26 \\
1,26\end{array}$ & 2,50 & 1,88 & 105,93 & 78,44 & 74,05 \\
\hline Almeria .. & 23,88 & 1,38 & 3,25 & 2,31 & 114,05 & 96,59 & 84,70 \\
\hline 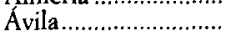 & 20,84 & 1,21 & 1,75 & 1,48 & 99,53 & 61,75 & 62,05 \\
\hline 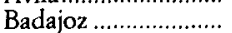 & 19,19 & 2,32 & 2,62 & 2,47 & 91,65 & 103,06 & 112,45 \\
\hline Barcelona .................... & 22,82 & 2,32 & 4,50 & 3,41 & 108,98 & 142,28 & 130,55 \\
\hline 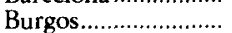 & 18,88 & 1,77 & 2,18 & 1,97 & 90,17 & 82,41 & 91,39 \\
\hline 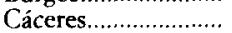 & 21,10 & 1,16 & 2,12 & 1,64 & 100,77 & 68,43 & 67,91 \\
\hline 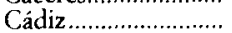 & 22,16 & 1,28 & 3,25 & 2,26 & 105,83 & 94,51 & 89,30 \\
\hline Castellón... & 21,33 & 1,34 & 2,87 & 2,10 & 101,87 & 87,83 & 86,22 \\
\hline 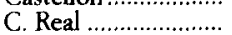 & 18,31 & 1,40 & 2,31 & 1,85 & 87,45 & 77,40 & 88,51 \\
\hline 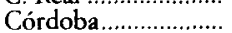 & 22,51 & 1,26 & 3,14 & 2,20 & 107,50 & 91,80 & 85,39 \\
\hline Coruña, La ................. & 18,58 & 1,25 & 3,00 & 2,13 & 88,74 & 88,67 & 99,92 \\
\hline Cuenca ......................... & 16,58 & 1,90 & 2,87 & 2,38 & 79,18 & 99,51 & 125,68 \\
\hline Gerona ....................... & 22,23 & 2,02 & 4,15 & 3,08 & 106,17 & 128,72 & 121,24 \\
\hline Granada ...................... & 20,50 & 1,87 & 3,00 & 2,43 & 97,90 & 101,60 & 103,77 \\
\hline 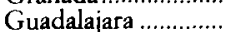 & 19,58 & 1,98 & 3,43 & 2,70 & 93,51 & 112,87 & 120,70 \\
\hline Guipúzcoa & 20,01 & 2,10 & 3,25 & 2,67 & 95,56 & 111,61 & 116,79 \\
\hline Huelva .................... & 23,52 & 2,56 & 3,56 & 3,06 & 112,33 & 127,68 & 113,67 \\
\hline Huesca ......................... & 22,31 & 1,77 & - & 1,77 & 106,55 & 73,85 & 69,31 \\
\hline 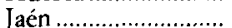 & 24,51 & 1,39 & 3,15 & 2,27 & 117,06 & 94,72 & 80,92 \\
\hline León ............................. & 18,62 & 0,80 & 2,75 & 1,77 & 88,93 & 74,06 & 83,28 \\
\hline 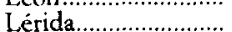 & 22,82 & 1,92 & 2,81 & 2,36 & 108,98 & 98,68 & 90,54 \\
\hline 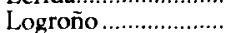 & 21,66 & 2,51 & 3,31 & 2,91 & 103,44 & 121,42 & 117,38 \\
\hline 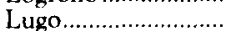 & 20,11 & 1,26 & 2,37 & 1,81 & 96,04 & 75,73 & 78,85 \\
\hline 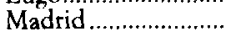 & 20,85 & 1,59 & 3,56 & 2,57 & 99,58 & 107,44 & 107,90 \\
\hline Málaga ...................... & 19,39 & 1,31 & 3,18 & 2,24 & 92,60 & 93,67 & 101,15 \\
\hline Murcia ....................... & 20,45 & 1,39 & 2,74 & 2,06 & 97,67 & 86,16 & 88,22 \\
\hline Navarra ........................ & 20,66 & 1,85 & 3,50 & 2,67 & 98,67 & 111,61 & 113,12 \\
\hline 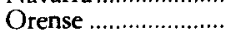 & 14,00 & 1,71 & 2,12 & 1,91 & 66,86 & 79,90 & 119,51 \\
\hline 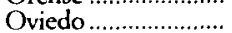 & 23,40 & 1,50 & 3,06 & 2,28 & 111,75 & 95,13 & 85,13 \\
\hline 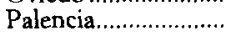 & 20,75 & 1,40 & 2,78 & 2,09 & 99,10 & 87,21 & 88,00 \\
\hline 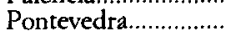 & 23,51 & 2,50 & - & 2,50 & 112,28 & 104,31 & 92,90 \\
\hline Salamanca .................. & 20,32 & 1,16 & 4,68 & 2,92 & 97,05 & 121,84 & 125,55 \\
\hline 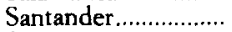 & 17,04 & 1,86 & 4,25 & 3,05 & 81,38 & 127,47 & 156,64 \\
\hline Segovia ....................... & 19,63 & 1,98 & 3,75 & 2,86 & 93.75 & 119,54 & 127,51 \\
\hline Sevilla ........................ & 23,11 & 1,67 & 3,50 & 2,58 & 110,37 & 107,86 & 97,73 \\
\hline Soria & 19,29 & 1,85 & 2,24 & 2,04 & 92,13 & 85,33 & 92,62 \\
\hline Tarragona.................. & 24,27 & 1,69 & 3,88 & 2,78 & 115,91 & 116,20 & 100,25 \\
\hline 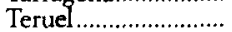 & 20,05 & 2,18 & 2,13 & 2,15 & 95,76 & 89,92 & 93,90 \\
\hline Toledo........................ & 23,45 & 1,66 & - & 1,66 & 111,99 & 69,26 & 61,85 \\
\hline Valencia .................... & 25,11 & 2,50 & 2,75 & 2,63 & 119,92 & 109,53 & 91,33 \\
\hline Valladolid............... & 20,48 & 2,21 & 2,63 & 2,42 & 97,81 & 100,97 & 103,24 \\
\hline 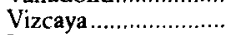 & 19,07 & 2,06 & 3,00 & 2,53 & 91,08 & 105,56 & 115,91 \\
\hline Zamora..................... & 20,38 & 1,58 & 3,75 & 2,66 & 9733 & 11120 & 114.25 \\
\hline 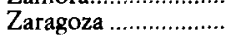 & 23,83 & 1,64 & 3,94 & 2,79 & 113,81 & 116,41 & 102,29 \\
\hline España ...................... & 20,939 & 1,712 & 3,081 & 2,397 & 100,00 & 100,00 & 100,00 \\
\hline Muestra....................... & 21,380 & 1,710 & 3,200 & 2,450 & 102,10 & 102,29 & 100,19 \\
\hline
\end{tabular}

(1): Jornal medio de peones agricolas en poblaciones de hasta 6.000 habitantes; (2): Jornal medio de los obreros fabriles e industriales en las capitales de provincia; (3): Media entre (1) y (2); (4): Cociente precio del trigo y media de España; (5): Cociente media de los jornales y media de España; (6): Cociente (4)/(5).

FUENTES: Precio del trigo, GEHR (1981), pp. 185-186; Jornales, Instituto Geográfico y Estadístico (1903), pp. XLVII-XIVIII. 
TABLA 3

Precios del pan, jornales diarios (en pesetas) y números indices en 1915

\begin{tabular}{|c|c|c|c|c|c|c|c|}
\hline & \multirow{2}{*}{ 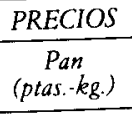 } & \multicolumn{3}{|c|}{ JORNALES } & \multicolumn{3}{|c|}{ INDICES (base, España = 100) } \\
\hline & & $\begin{array}{c}A 1915 \\
(1)\end{array}$ & $\begin{array}{c}U 1915 \\
(2)\end{array}$ & $\begin{array}{c}\text { Media } \\
(3)\end{array}$ & $\begin{array}{c}\text { Precios } \\
(4)\end{array}$ & $\begin{array}{c}\text { Jornales } \\
(5)\end{array}$ & $\begin{array}{c}\text { Salario real } \\
(6)\end{array}$ \\
\hline Álava ....... & 0,44 & 2,65 & 2,60 & 2,62 & 100,68 & 106,98 & 106,25 \\
\hline Albacete .................. & 0,39 & 2,02 & 2,50 & 2,26 & 89,24 & 92,10 & 103,20 \\
\hline Alicante.................... & 0,45 & 1,84 & 3,20 & 2,52 & 102,97 & 102,70 & 99,73 \\
\hline Almería .................... & 0,40 & 1,80 & 2,40 & 2,10 & 91,53 & 85,58 & 93,50 \\
\hline 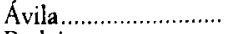 & 0,44 & 2,02 & 2,50 & 2,26 & 100,68 & 92,10 & 91,48 \\
\hline Badajoz ..................... & 0,40 & 1,80 & 2,00 & 1,90 & 91,53 & 77,43 & 84,60 \\
\hline Barcelona .................. & 0,49 & 2,80 & 3,60 & 3,20 & 112,12 & 130,41 & 116,31 \\
\hline 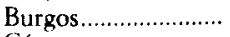 & 0,43 & 2,31 & 2,70 & 2,50 & 98,39 & 102,09 & 103,75 \\
\hline Cáceres............................ & 0,36 & 1.51 & 2,50 & 2,00 & 82,38 & 81,71 & 99,19 \\
\hline Cádiz........ & 0,46 & 1,84 & 3,40 & 2,62 & 105,26 & 106,77 & 101,44 \\
\hline Castellón ................... & 0,43 & 1,65 & 3,10 & 2,37 & 98,39 & 96,79 & 98,37 \\
\hline 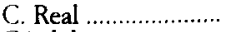 & 0,40 & 1,86 & 3,00 & 2,43 & 91,53 & 99,03 & 108,19 \\
\hline Córdoba...... & 0,42 & 1,84 & 2,70 & 2,27 & 96,11 & 92,51 & 96,26 \\
\hline Coruña, La ............... & 0,50 & 2,02 & 3,30 & 2,66 & 114,41 & 108,40 & 94,75 \\
\hline Cuenca ..................... & 0,37 & 1,83 & 2,30 & 2,06 & 84,66 & 84,15 & 99,40 \\
\hline 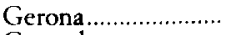 & 0,46 & 2,69 & 3,10 & 2,89 & 105,26 & 117,98 & 112,09 \\
\hline Granada ...................... & 0,42 & 1,58 & 2,70 & 2,14 & 96,11 & 87,21 & 90,74 \\
\hline Guadalajara ............. & 0,39 & 1,74 & 2,80 & 2,27 & 89,24 & 92,51 & 103,66 \\
\hline Guipúzcoa .............. & 0,46 & 2,75 & 3,90 & 3,32 & 105,26 & 135,50 & 128,73 \\
\hline Huelva ......... & 0.49 & 2,00 & 3,30 & 2,65 & 112,12 & 107,99 & 96,32 \\
\hline Huesca.. & 0,44 & 2,44 & 2,50 & 2,47 & 100,68 & 100,66 & 99,98 \\
\hline Jaén ........... & 0,40 & 1,83 & 2,50 & 2,16 & 91,53 & 88,23 & 96,40 \\
\hline León.......... & 0,38 & 1,96 & 2,60 & 2,28 & 86,95 & 92,92 & 106,86 \\
\hline Lérida... & $.0,46$ & 2,41 & 3,20 & 2,80 & 105,26 & 114,31 & 108,60 \\
\hline Logroño.... & 0,45 & 2,07 & 2,70 & 2,38 & 102,97 & 97,19 & 94,39 \\
\hline Lugo............................... & 0,52 & 2,11 & 2,40 & 2,25 & 118,99 & 91,90 & 77,23 \\
\hline Madrid ..................... & 0,44 & 2,12 & 4,20 & 3,16 & 100.68 & 128,78 & 127,91 \\
\hline Málaga ..................... & 0,47 & 1,77 & 3,00 & 2,38 & 107,55 & 97,19 & 90,37 \\
\hline Murcia ...................... & 0,42 & 1,79 & 2,70 & 2,24 & 96,11 & 91,49 & 95,20 \\
\hline Navarra.................... & 0,42 & 2,54 & 2,70 & 2,62 & 96,11 & 106,77 & 111,10 \\
\hline Orense ..................... & 0,50 & 2,12 & 3,20 & 2,66 & 114,41 & 108,40 & 94,75 \\
\hline Oviedo ......... & 0,52 & 2,55 & 2,90 & 2,72 & 118,99 & 111,05 & 93,33 \\
\hline Palencia.................... & 0,42 & 1,78 & 3,20 & 2,49 & 96,11 & 101,47 & 105,59 \\
\hline Pontevedra... & 0,49 & 2,01 & 2,30 & 2,15 & 112,12 & 87,82 & 78,33 \\
\hline Salamanca ................ & 0,41 & 2,08 & 3,30 & 2,69 & 93,82 & 109,62 & 116,85 \\
\hline Santander................. & 0,47 & 2,38 & 3,30 & 2,84 & 107,55 & 115,74 & 107,62 \\
\hline 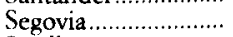 & 0,38 & 1,85 & 2,00 & 1,92 & 86,95 & 78,45 & 90,22 \\
\hline 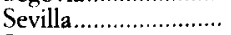 & 0,46 & 2,17 & 3,20 & 2,68 & 105,26 & 109,42 & 103,95 \\
\hline 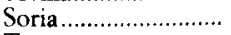 & 0,40 & 1,77 & 2,20 & 1,98 & 91,53 & 80,89 & 88,38 \\
\hline Tarragona.................. & 0,46 & 2,03 & 3,50 & 2,76 & 105,26 & 112,68 & 107,05 \\
\hline Teruel ........................ & 0,42 & 1,92 & 2,50 & 2,21 & 96,11 & 90,06 & 93,71 \\
\hline Toledo...................... & 0,41 & 1,84 & 2,60 & 2,22 & 93,82 & 90,47 & 96,43 \\
\hline Valencia & 0,43 & 1,98 & 3,00 & 2,49 & 98,39 & 101,47 & 103,13 \\
\hline Valladolid... & 0,41 & 1,85 & 2,10 & 1,97 & 93,82 & 80,49 & 85,79 \\
\hline 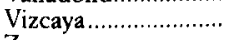 & 0,47 & 2,66 & 2,90 & 2,78 & 107,55 & 113,29 & 105,34 \\
\hline 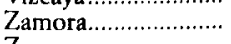 & 0,44 & 1,82 & 2,80 & 2,31 & 100,68 & 94,14 & 93,50 \\
\hline & 0,45 & 2,36 & 2,80 & 2,58 & 102,97 & 105,14 & 102,11 \\
\hline 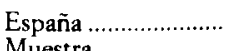 & $\begin{array}{l}0,437 \\
0,440\end{array}$ & 2,059 & $\begin{array}{l}2,849 \\
2,980\end{array}$ & $\begin{array}{l}2,454 \\
2,540\end{array}$ & $\begin{array}{l}100,00 \\
100,68\end{array}$ & $\begin{array}{l}100,00 \\
103,73\end{array}$ & $\begin{array}{l}100,00 \\
102,78\end{array}$ \\
\hline Muestra..................... & 0,440 & 2,110 & 2,98 & & & & \\
\hline
\end{tabular}
(1): Jornal medio de peones agrícolas; (2): Salarios/hora de peones ordinarios, multiplicador 10; (3): Media de España; (6): Cociente (4)/(5).

FUENTES: Precio del pan, Boletín del Instituto de Reformas Sociales, tomo XII; Jornales agrícolas, Instituto Geográfico y Estadistico (1916), pp. 244-245; Jornales no agricolas, Dirección General del Trabajo (1931), p. XXXVI 
TABLA 4

Precios del pan, jornales diarios (en pesetas) y números indices en 1930

\begin{tabular}{|c|c|c|c|c|c|c|c|}
\hline & \multirow{2}{*}{ 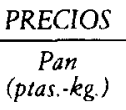 } & \multicolumn{3}{|c|}{ JORNALES } & \multicolumn{3}{|c|}{ INDICES (base, España = 100) } \\
\hline & & $\begin{array}{c}A 1930 \\
(1)\end{array}$ & $\begin{array}{c}U 1930 \\
(2)\end{array}$ & $\begin{array}{c}\text { Media } \\
\text { (3) }\end{array}$ & $\begin{array}{c}\text { Precios } \\
(4)\end{array}$ & $\begin{array}{c}\text { Jornales } \\
\text { (5) }\end{array}$ & $\begin{array}{c}\text { Salario real } \\
(6)\end{array}$ \\
\hline Álava ...... & 0,59 & 3,75 & 4,72 & 4,23 & 95,00 & 84,28 & 88,72 \\
\hline Albacete................... & 0,61 & 4,50 & 3,84 & 4,17 & 98,22 & 82,99 & 84,50 \\
\hline Alicante................... & 0,65 & 5,25 & 4,56 & 4,90 & 104,66 & 97,62 & 93,27 \\
\hline 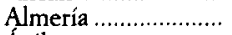 & 0,61 & 5,25 & 3,28 & 4,26 & 98,22 & 84,88 & 86,42 \\
\hline Ávila ......................... & 0,60 & 3,88 & 3,92 & 3,90 & 96,61 & 77,62 & 80,34 \\
\hline Badajoz ................... & 0,58 & 4,25 & 3,84 & 4,04 & 93,39 & 80,50 & 86,20 \\
\hline Barcelona ................. & 0,70 & 8,50 & 6,80 & 7,65 & 112,71 & 152,25 & 135,08 \\
\hline Burgos........................ & 0,59 & 5,00 & 4,72 & 4,86 & 95,00 & 96,72 & 101.81 \\
\hline Cáceres....................... & 0,56 & 3,25 & 4,00 & 3,63 & 90,17 & 72,14 & 80,01 \\
\hline Cádiz ...................... & 0,60 & 5,43 & 4,80 & 5,11 & 96,61 & 101,80 & 105,37 \\
\hline Castellón .................. & 0,65 & 8,00 & 4,08 & 6,04 & 104,66 & 120,21 & 114,85 \\
\hline C. Real ..... & 0,57 & 5,50 & 4,24 & 4,87 & 91,78 & 96,92 & 105,60 \\
\hline Córdoba.................... & 0,58 & 5,00 & 4,24 & 4,62 & 93,39 & 91,95 & 98.46 \\
\hline Coruña, La............... & 0,70 & 5,50 & 5,12 & 5,31 & 112,71 & 105,68 & 93,76 \\
\hline Cuenca ..................... & 0,55 & 4,50 & 3,60 & 4,05 & 88,56 & 80,60 & 91,02 \\
\hline Gerona ...................... & 0,70 & 6,50 & 4.60 & 5,55 & 112,71 & 110,45 & 98,00 \\
\hline Granada ..................... & 0,58 & 4,25 & 3,92 & 4,09 & 93,39 & 81,30 & 87,05 \\
\hline Guadalajara .............. & 0,60 & 4,25 & 3,84 & 4,04 & 96,61 & 80,50 & 83.33 \\
\hline Guipúzcoa .............. & 0,64 & 5,75 & 5,92 & 5,84 & 103,05 & 116,13 & 112,69 \\
\hline Huelva ...................... & 0,65 & 4,75 & 4,48 & 4,61 & 104,66 & 91,85 & 87.76 \\
\hline Huesca ..................... & 0,55 & 5,25 & 5,44 & 5,34 & 88,56 & 106,37 & 120,12 \\
\hline 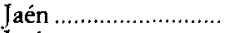 & 0,53 & 5,25 & 3,84 & 4.54 & 85,34 & 90,45 & 105,99 \\
\hline 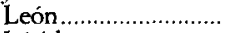 & 0,61 & 5,50 & 5,36 & 5.43 & 98,22 & 108,07 & 110,03 \\
\hline Lérida ......................... & 0,65 & 8,50 & 4,80 & 6,65 & 104,66 & 132.35 & 126,45 \\
\hline Logroño ...................... & 0,60 & 5,50 & 4,88 & 5,19 & 96,61 & 103,29 & 106,92 \\
\hline 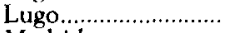 & 0,65 & 5,00 & 4,16 & 4,58 & 104,66 & 91,15 & 87,09 \\
\hline Madrid .................... & 0,65 & 6,50 & 7,60 & 7,05 & 104,66 & 140,31 & 134,06 \\
\hline Málaga .................... & 0,62 & 4,50 & 5,04 & 4,77 & 99,83 & 94,93 & 95,09 \\
\hline Murcia .................... & 0,60 & 4,75 & 3.76 & 4,25 & 96,61 & 84.68 & 87,65 \\
\hline Navarra .................... & 0,62 & 7,75 & 5,68 & 6,71 & 99,83 & 133,64 & 133,87 \\
\hline Orense ..................... & 0,67 & 5,00 & 4,56 & 4,78 & 107,88 & 95,13 & 88,18 \\
\hline Oviedo .......................... & 0,75 & 6,75 & 7,04 & 6,89 & 120,76 & 137,22 & 113,63 \\
\hline Palencia .................... & 0,59 & 5,00 & 4,80 & 4,90 & 95,00 & 97,52 & 102,65 \\
\hline Pontevedra............... & 0,70 & 4,25 & 4,32 & 4,28 & 112,71 & 85,28 & 75,66 \\
\hline Salamanca ................ & 0,60 & 2,50 & 4,80 & 3,65 & 96,61 & 72,64 & 75,19 \\
\hline Santander.................... & 0,70 & 5,50 & 6,00 & 5,75 & 112,71 & 114,44 & 101,53 \\
\hline Segovia .......................... & 0,60 & 5,25 & 4,00 & 4,63 & 96,61 & 92,05 & 95,28 \\
\hline 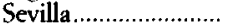 & 0,59 & 7,00 & 4,88 & 5,94 & 95,00 & 118,22 & 124,44 \\
\hline Soria ............................. & 0,58 & 5,50 & 4,32 & 4,91 & 93,39 & 97,72 & 104,64 \\
\hline Tarragona...................... & 0,70 & 7,50 & 5,04 & 6,27 & 112,71 & 124,78 & 110,71 \\
\hline Teruel ....................... & 0,60 & 5,00 & 4,56 & 4,78 & 96,61 & 95,13 & 98,47 \\
\hline Toledo........................ & 0,60 & 4,13 & 4,00 & 4,06 & 96,61 & 80,90 & 83,74 \\
\hline Valencia ................... & 0,65 & 4,50 & 4,64 & 4,57 & 104,66 & 90,95 & 86,90 \\
\hline Valladolid ................. & 0,62 & 4,00 & 4,64 & 4,32 & 99,83 & 85,98 & 86,12 \\
\hline Vizcaya ...................... & 0,65 & 6,50 & 6,80 & 6,65 & 104,66 & 132,35 & 126,45 \\
\hline Zamora...................... & 0,60 & 3,00 & 3,52 & 3,26 & 96,61 & 64,88 & 67,16 \\
\hline Zaragoza ................... & 0,60 & 7,50 & 4,88 & 6,19 & 96,61 & 123,19 & 127,52 \\
\hline España ..................... & 0,621 & 5,329 & 4,721 & 5,025 & 100,00 & 100,00 & 100,00 \\
\hline Muestra................... & 0,630 & 5,840 & 5,240 & 5,540 & 100,90 & 110,30 & 108,78 \\
\hline
\end{tabular}

(1): Jornal medio de peones agrícolas; (2): Salarios/hora de peones ordinarios, multiplicador 8; (3): Media entre (1) y (2); (4): Cociente precio del pan y media de España; (5): Cociente media de los jornales y media de España; (6): Cociente (4)/(5).

FUENTES: Precio del pan, Boletin del Ministerio de Trabajo (1930), tomo I, pp. 106-107; Jornales agrícolas, Instituto Geografico y Estadístico (1932), p. 549; Jornales no agrícolas, Dirección General del Trabajo (1931), p. XXXVI. 


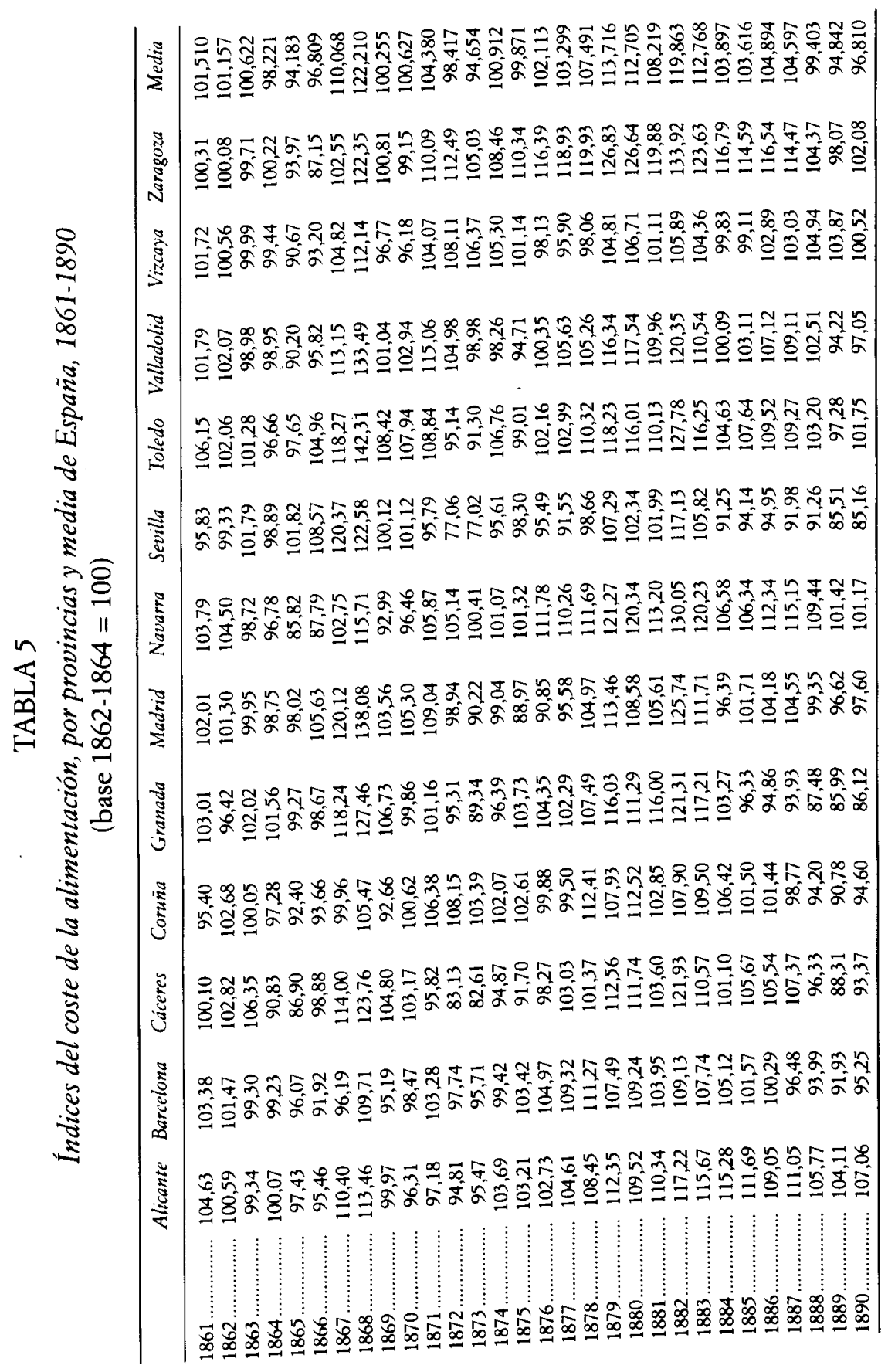




\section{TABLA 6}

Indices del coste de la alimentación, 1889-1909, Madrid, Valladolid y Vizcaya (base 1900-1902 = 100)

\begin{tabular}{|c|c|c|c|c|}
\hline & Madrid & Valladolid & Vizcaya & Media \\
\hline $1889 \ldots \ldots$. & 98,68 & 81,26 & 121,53 & 99,83 \\
\hline 1890 & 92,83 & 80,57 & 113,70 & 95,70 \\
\hline 1891 & 96,84 & 84,74 & 104,62 & 95,40 \\
\hline $1892 \ldots \ldots$ & 100,71 & 90,36 & 107,76 & 99,61 \\
\hline 1893 & 106,04 & 89,16 & 102,31 & 99,17 \\
\hline 1894 & 100,42 & 88,09 & 104,57 & 97,69 \\
\hline 1895 & 99,58 & 82,21 & 103,72 & 95,17 \\
\hline 1896 & 96,92 & 83,35 & 120,60 & 100,29 \\
\hline 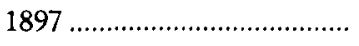 & 95,24 & 91,09 & 127,83 & 104,72 \\
\hline 1898 & 103,24 & 95,64 & 134,62 & 111,17 \\
\hline 1899 & 95,13 & 100,21 & 118,17 & 104,50 \\
\hline 1900 & 99,95 & 101,93 & 97,24 & 99,94 \\
\hline 1901 & 100,42 & 99,72 & 99,98 & 100,04 \\
\hline 1902 & 99,63 & 98,36 & 102,07 & 100,02 \\
\hline 1903 & 102,30 & 99,36 & 112,29 & 104,65 \\
\hline $1904 \ldots \ldots \ldots \ldots \ldots \ldots \ldots$ & 100,23 & 103,70 & 117,52 & 107,15 \\
\hline 1905 & 107,45 & 104,59 & 117,52 & 109,85 \\
\hline 1906 & 100,78 & 101,15 & 119,51 & 107,15 \\
\hline 1907 & 109,74 & 99,42 & 114,96 & 108,04 \\
\hline 1908 & 108,88 & 97,73 & 114,37 & 106,99 \\
\hline 1909 & 104,15 & 102,58 & 113,03 & 106,59 \\
\hline
\end{tabular}




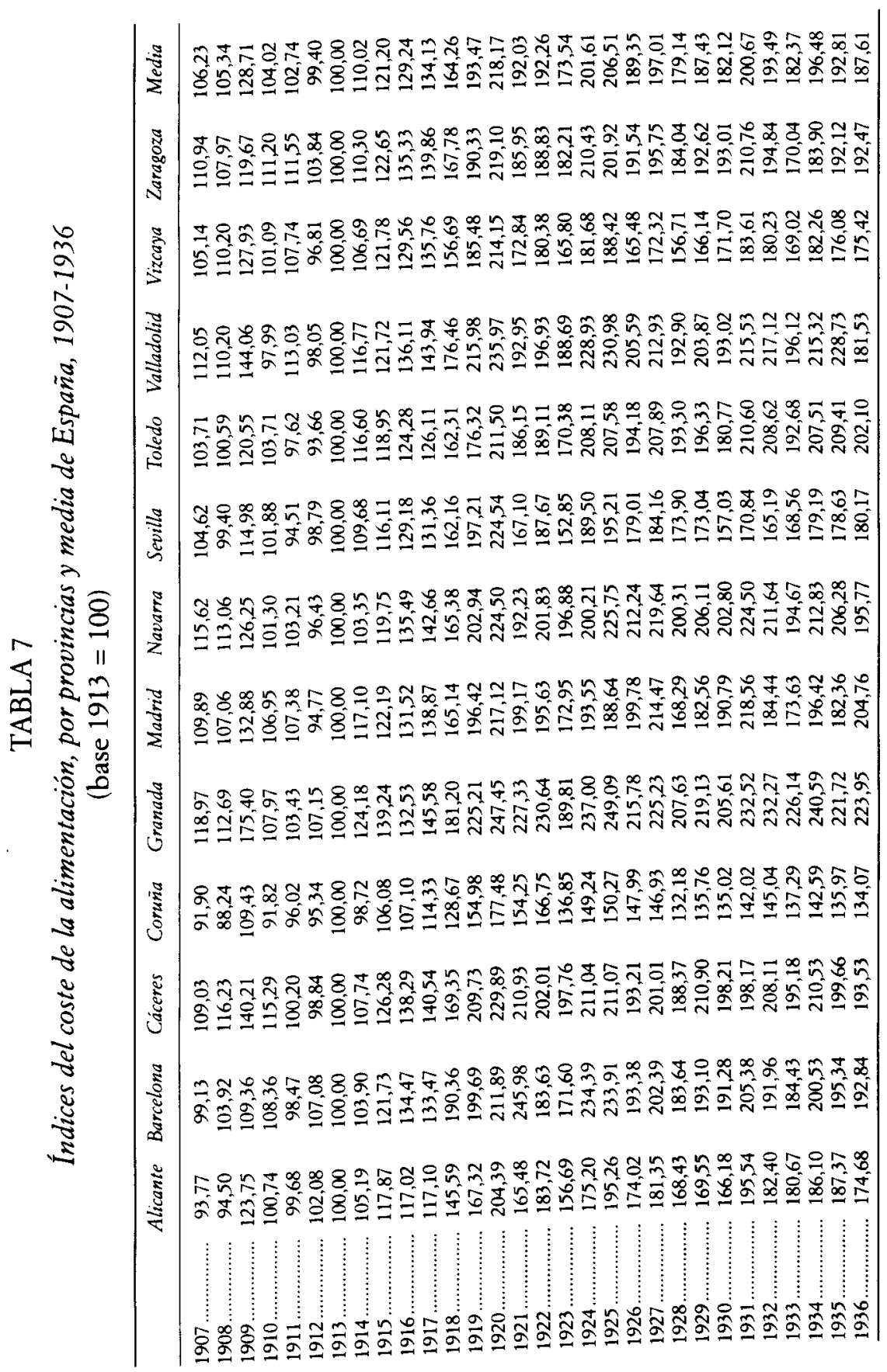


TABLA 8

Indice del coste de la vida en España, 1861-1936 (base $1913=100$ )

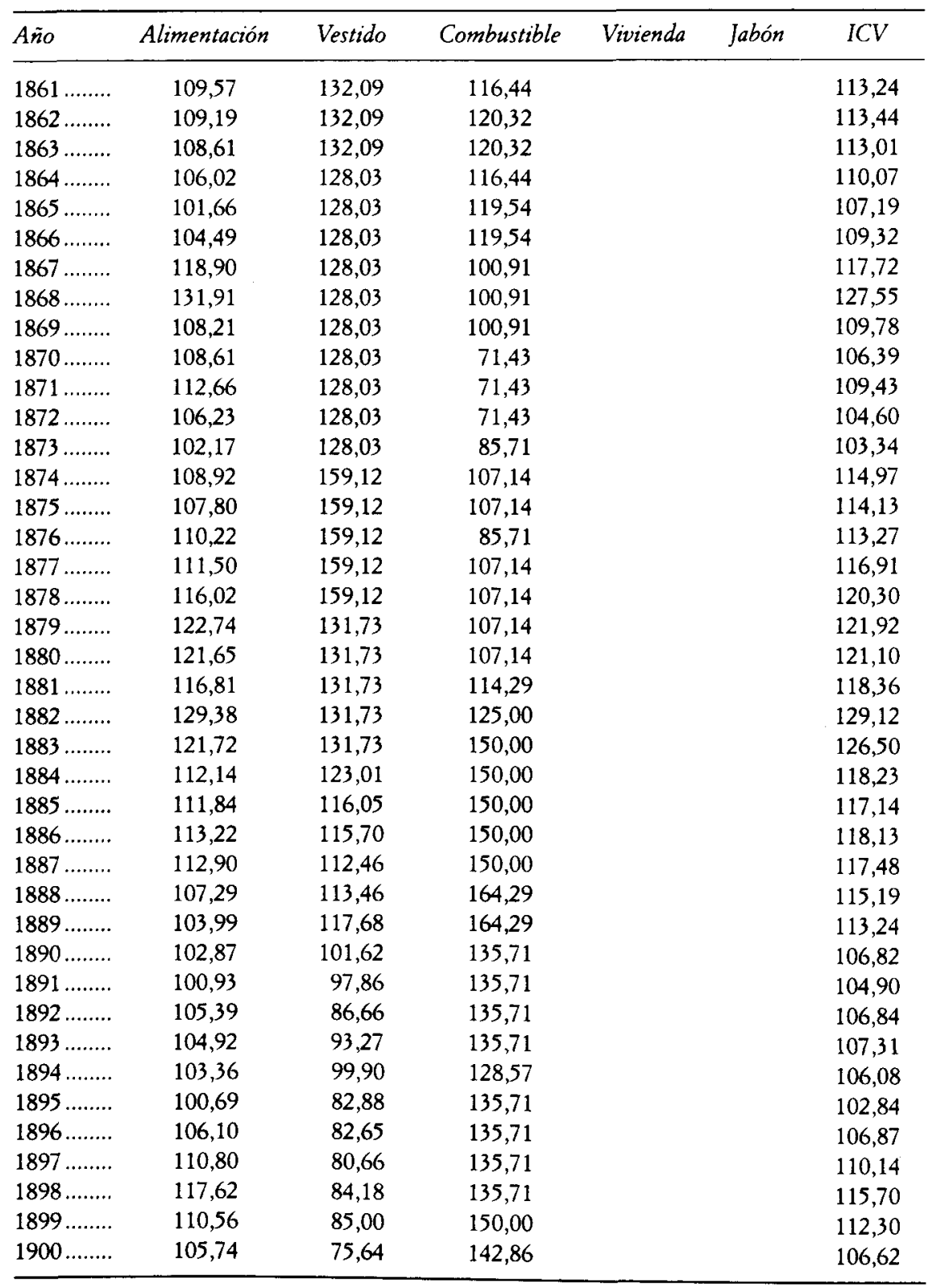


TABLA 8 (continuación)

\begin{tabular}{|c|c|c|c|c|c|c|}
\hline Año & Alimentación & Vestido & Combustible & Vivienda & Jabón & $I C V$ \\
\hline $1901 \ldots \ldots .$. & 105,84 & 73,30 & 135,71 & & & 105,51 \\
\hline $1902 \ldots \ldots$. & 105,82 & 75,00 & 135,71 & 101,94 & & 105,34 \\
\hline $1903 \ldots \ldots$ & 110,72 & 77,26 & 135,71 & 101,94 & & 109,00 \\
\hline $1904 \ldots \ldots . .$. & 113,36 & 64,80 & 135,71 & 101,94 & & 109,60 \\
\hline $1905 \ldots \ldots$ & 116,23 & 85,12 & 135,71 & 101,94 & & 113,64 \\
\hline $1906 \ldots \ldots$ & 113,36 & 77,46 & 135,71 & 101,94 & & 110,87 \\
\hline $1907 \ldots \ldots$. & 110,27 & 85,46 & 97,86 & 101,94 & & 105,71 \\
\hline $1908 \ldots \ldots \ldots$ & 109,27 & 79,07 & 94,17 & 101,94 & & 104,01 \\
\hline $1909 \ldots \ldots$. & 120,74 & 97,25 & 108,99 & 100,97 & 114,18 & 115,50 \\
\hline $1910 \ldots \ldots$ & 104,02 & 99,17 & 101,60 & 100,00 & 96,51 & 102,64 \\
\hline $1911 \ldots \ldots$. & 102,74 & 101,09 & 101,91 & 100,00 & 99,45 & 102,09 \\
\hline $1912 \ldots \ldots$ & 99,40 & 101,85 & 100,63 & 100,00 & 98,15 & 99,71 \\
\hline $1913 \ldots \ldots$. & 100,00 & 100,00 & 100,00 & 100,00 & 100,00 & 100,00 \\
\hline $1914 \ldots \ldots$ & 110,02 & 98,94 & 104,48 & 99,03 & 100,87 & 107,08 \\
\hline $1915 \ldots \ldots .$. & 121,20 & 99,52 & 110,36 & 98,06 & 100,55 & 115,14 \\
\hline $1916 \ldots \ldots$ & 129,24 & 109,84 & 119,54 & 98,06 & 104,69 & 122,47 \\
\hline $1917 \ldots \ldots$. & 134,13 & 84,39 & 109,26 & 92,23 & 138,17 & 123,93 \\
\hline $1918 \ldots \ldots$. & 164,26 & 95,39 & 129,82 & 83,98 & 189,20 & 148,87 \\
\hline $1919 \ldots \ldots$. & 193,47 & 89,89 & 141,68 & 83,98 & 189,97 & 169,40 \\
\hline $1920 \ldots \ldots$. & 218,17 & 91,26 & 154,71 & 122,33 & 202,07 & 191,91 \\
\hline $1921 \ldots \ldots$. & 192,03 & 89,20 & 140,61 & 128,16 & 192,69 & 172,82 \\
\hline $1922 \ldots \ldots$. & 192,26 & 94,01 & 143,13 & - 169,17 & 176,55 & 176,88 \\
\hline $1923 \ldots \ldots$. & 173,54 & 96,24 & 134,89 & 210,19 & 158,34 & 166,78 \\
\hline $1924 \ldots \ldots .$. & 201,61 & 90,70 & 146,15 & 210,19 & 153,54 & 186,20 \\
\hline $1925 \ldots \ldots$. & 206,51 & 109,29 & 157,90 & 210,19 & 152,78 & 192,04 \\
\hline $1926 \ldots \ldots$ & 189,35 & 134,44 & 161,89 & 210,19 & 146,67 & 182,44 \\
\hline $1927 \ldots \ldots .$. & 197,01 & 152,56 & 192,86 & 281,07 & 147,66 & 198,29 \\
\hline $1928 \ldots \ldots .$. & 179,14 & 181,91 & 192,86 & 291,26 & 137,84 & 189,25 \\
\hline $1929 \ldots \ldots .$. & 187,43 & 212,61 & 192,86 & 290,05 & 140,35 & 198,12 \\
\hline $1930 \ldots \ldots$ & 182,12 & 153,81 & 192,86 & 288,83 & 132,93 & 188,04 \\
\hline $1931 \ldots \ldots$. & 200,67 & 186,54 & 192,86 & 288,83 & 131,84 & 204,24 \\
\hline $1932 \ldots \ldots$ & 193,49 & 238,49 & 192,86 & & 132,39 & 197,12 \\
\hline $1933 \ldots \ldots$ & 182,37 & 226,64 & 192,86 & & 126,72 & 187,28 \\
\hline $1934 \ldots \ldots$. & 196,48 & 213,68 & 178,57 & & 127,37 & 193,81 \\
\hline $1935 \ldots \ldots$ & 192,81 & 209,14 & 178,57 & & 127,81 & 190,58 \\
\hline $1936 \ldots \ldots$. & 187,61 & 205,70 & 178,57 & & 137,62 & 186,92 \\
\hline
\end{tabular}

